\title{
Efficient and Eco-friendly route for the Solvent Free Synthesis of 4-Alkoxy-5H- chromen[2,3-d]pyrimidines Using Phosphonic Acid Functionalized KIT-6 Confined Ionic Liquid as Recoverable Catalyst
}

Nastaran Karimi, Hoda Yahyavi, Leila Ma'mani, Mohammad Mahdavi, Alireza Foroumadi, and Abbas Shafiee*

\section{Contents:}

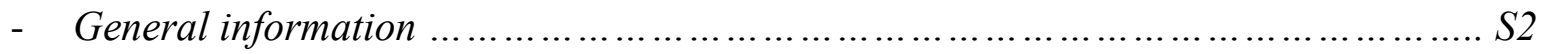

- Preparation of catalyst......................................................... $S 2$

- Synthesis of amine functionalized mesoporous

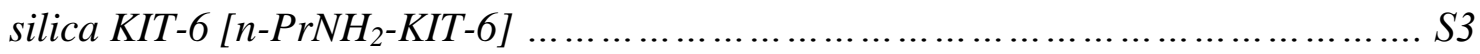

- Synthesis of phosphonic acid functionalized Ia3d mesoporous silica nanoparticles [Phosphonic acid@ KIT-6] ..............................S3

- Synthesis of phosphonic acid functionalized Ia3d mesoporous silica nanoparticles confined ionic liquid, IL@ [Phosphonic acid@KIT-6] .............S3

- General procedure for the synthesis of 4-methoxy-5H-chromen[2,3-d] pyrimidine using IL@ [Phosphonic acid@KIT-6] as catalyst............................. S4

- The recovery of IL@ [Phosphonic acid@KIT-6].......................................... S4

- The Characterization of the Nanocatalyst ........................................ S5

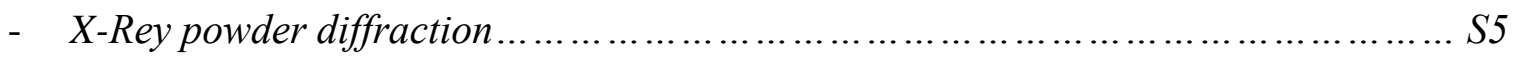

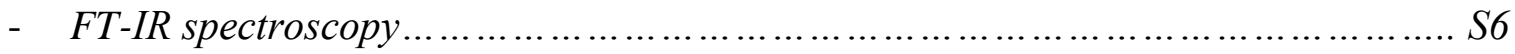

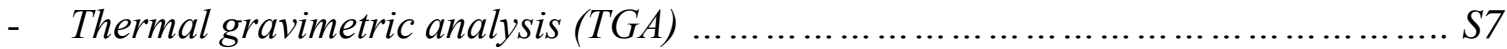

- $\quad N_{2}$ sorption isotherm experiment ............................................... $S 8$

- ${ }^{1}$ H NMR and ${ }^{13}$ C NMR data of the products ......................................... S9

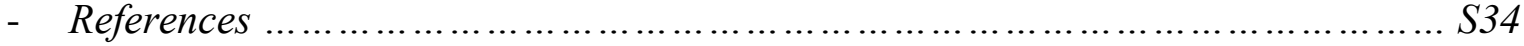




\section{- General information}

All chemicals are analytical-grade reagents and used without further purification and all solvents were purified and dried using standard procedures. ${ }^{1} \mathrm{H}$ and ${ }^{13} \mathrm{C}$ NMR was obtained on a 400 or $500 \mathrm{MHz}$ Bruker Avance instrument using $\mathrm{CDCl}_{3}$ as solvent and TMS as internal standard. TLC was conducted on silica gel $250 \mathrm{~m}$, F254 plates. Melting points were measured on a Kofler hot stage apparatus and uncorrected. The powder XRD spectrum was recorded at r.t. with a Philips 1710 diffractometer using $\mathrm{Cu} \mathrm{K} \alpha\left(\lambda=1.54056 \mathrm{~A}^{\circ}\right)$ in $\mathrm{Bragg}-$ Brentano geometry $(\theta-2 \theta)$. The morphologies of the products were observed using SEM (Hitachi S-4800 II, Japan) equipped with energy dispersive X-ray spectroscopy. The IR spectra were taken using Nicolet FT-IR Magna 550 spectrographs (KBr disks). The surface areas were calculated by BET method and the pore size distributions were calculated from the adsorption branch of the isotherms using BJH method.

- Preparation of the catalyst

- Synthesis of amine functionalized mesoporous silica KIT-6, [n-PrNH $\left.{ }_{2}-\mathrm{KIT}-6\right]$

The amine functionalized mesoporous silica KIT-6 ([n- $\left.\left.\mathrm{PrNH}_{2}-\mathrm{KIT}-6\right]\right)$, was prepared according to the procedure reported in literature. ${ }^{[1]}$ Typically, $4.0 \mathrm{~g}$ of $n$ - $\mathrm{BuOH}$ was added to $4.0 \mathrm{~g}$ of P123 dissolved in $144 \mathrm{~g}$ of DI water and $7.9 \mathrm{~g}$ of $\mathrm{HCl}$ (35 wt. \%). After $1 \mathrm{~h}$ stirring at $35{ }^{\circ} \mathrm{C}, 8.6 \mathrm{~g}$ of TEOS was added to the clear solution, and the mixture was left under vigorous stirring at the same temperature for $24 \mathrm{~h}$. Then the reaction temperature was raised to $100{ }^{\circ} \mathrm{C}$ and aged under static condition for $24 \mathrm{~h}$. The white product was filtered off without washing and dried under vacuum at $100{ }^{\circ} \mathrm{C}$ overnight. Template material was removed by a brief $\mathrm{EtOH} / \mathrm{HCl}$ washing after calcination at $550{ }^{\circ} \mathrm{C}$ for $6 \mathrm{~h}$. 
Then, the as-synthesized KIT-6 (1g) and aminopropyl trimethoxysilane (APTMS) (4 mmol) were suspended in $30 \mathrm{~mL}$ toluene and heated at reflux overnight. Finally, the suspension was filtered off and washed several times with acetone, followed by drying under vacuum exhaustively. This support was referred to as aminopropyl-functionalized mesoporous silica [n-PrNH ${ }_{2}$-KIT-6].

- Synthesis of phosphonic acid functionalized Ia3d mesoporous silica nanoparticles [Phosphonic acid@KIT-6]

[n-PrNH$\left.{ }_{2}-\mathrm{KIT}-6\right]$ (0.2 mol amine group), crystalline phosphorous acid $(0.4 \mathrm{~mol})$, and conc. $\mathrm{HCl}(0.6 \mathrm{~mol})$ were dissolved in $200 \mathrm{~mL}$ water, and the mixture was refluxed in a three-necked flask fitted with thermometer, condenser, and dropping funnel. During of $1 \mathrm{~h}$, $60 \mathrm{~mL}$ of a $40 \%(\mathrm{w} / \mathrm{v})$ aqueous formaldehyde solution was added drop-wise, and the reaction was refluxed for 1 hour. The solvent was evaporated, and the concentrated residue was neutralized with conc. ammonia solution. Finally, the obtained solid was filtered off and washed with hot dry $\mathrm{MeOH}$ for $12 \mathrm{~h}$ in a continuous extraction apparatus (Soxhelet) and then dried at $100{ }^{\circ} \mathrm{C}$ overnight to furnish the corresponding surface bound phosphonic acid [Phosphonic acid@KIT-6].

- Synthesis of phosphonic acid functionalized Ia3d mesoporous silica nanoparticles confined ionic liquid, IL@[Phosphonic acid@KIT-6]

[Phosphonic acid@KIT-6] (1 g) was added to a solution of 1-butyl-3-methylimidazolium tetrafluoroborate $(3 \mathrm{~mL})$ in dry acetone $(50 \mathrm{~mL})$. The reaction mixture was stirred at room temperature overnight. After stirring, acetone was removed under reduced pressure. The 
resulting solid was then dried at $70{ }^{\circ} \mathrm{C}$ under vacuum for $24 \mathrm{~h}$, to achieve the designed catalyst IL@[KIT-6-Phosphonic acid].

- General procedure for the synthesis of 4-methoxy-5H-chromen[2,3-d]pyrimidine using IL@[KIT-6-Phosphonic acid] as catalyst

IL@[Phosphonic acid@KIT-6] catalyst (2 mol \%) was added gently to a magnetically stirred mixture of iminocoumarine 3(a-c) $(1 \mathrm{mmol})$, salicylaldehyde $\mathbf{1}(\mathbf{a}-\mathbf{c})(1 \mathrm{mmol})$, and alcohol 4(a-e) $(1.5 \mathrm{mmol})$. The reaction mixture was stirred for $40 \mathrm{~min}$ at room temperature. The progress of the reaction was monitored by TLC. When the spot for the product $(\mathrm{Rf} \approx 0.8$ in silica gel, EtOAc: n-hexane (1:5) was visible, the catalyst was separated by filtration or centrifuging, and the final product was purified by column chromatography using EtOAc: nhexane 1: 6 as eluent and recrystallized from $\mathrm{EtOH}$.

- The recovery of IL@[Phosphonicacid@KIT-6]

After the completion of the aforementioned reaction over the IL@[KIT-6-Phosphonic acid] catalyst, the resulting mixture was filtered and washed with EtOH and EtOAc thoroughly. The separated catalyst was then successfully recovered and reused 5 times under the same reaction condition. 
- The Characterization of the Nanocatalyst

\section{- X-Rey powder diffraction}

The appeared reflections at $2 \theta=0.87^{\circ}, 0.97^{\circ}$, and $1.7^{\circ}$ are indicating 211,220 , and 332 reflections, respectively (Figure S1), which clearly can be indexed to the reflections of the expected $3 D$ cubic symmetry of the space group Ia3d. Except for the decrease in peak intensity, the positions of peaks remained virtually fixed, and no significant changes were observed during the post modification process. These confirm that the functionalization mainly accomplished inside of the pores and the retention of the mesoporous structure of KIT-6, after the functionalization.

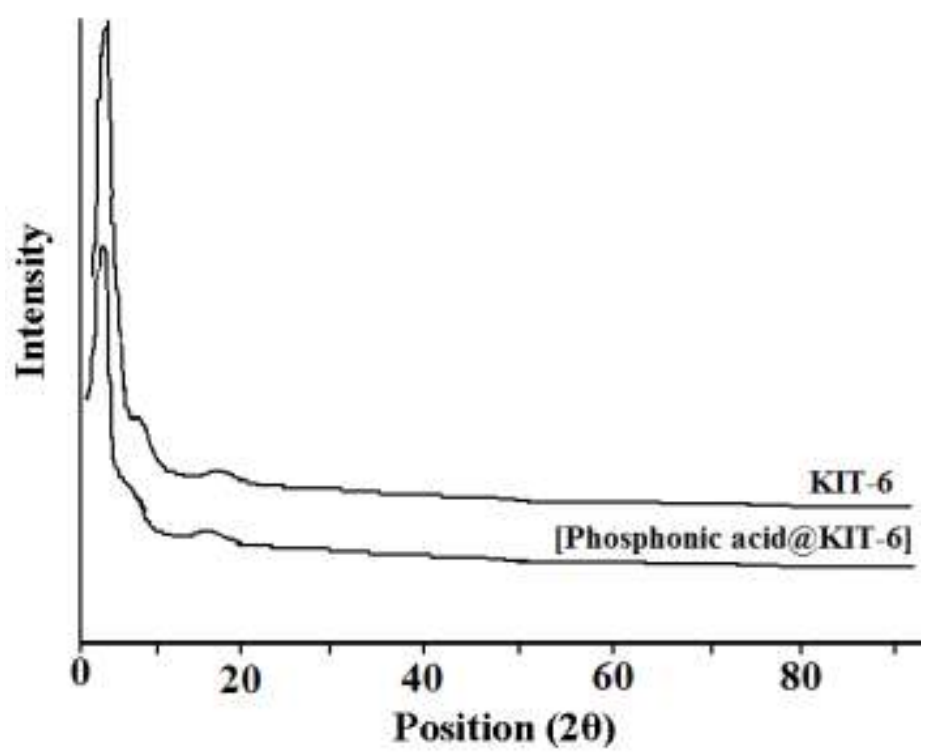

Figure S1. The XRD patterns of KIT-6 and [Phosphonic acid@KIT-6] 


\section{- FT-IR spectroscopy}

The presence of the aminopropyl-group and phosphonic acid on the mesopores surface was confirmed by FT-IR spectroscopy (Figure S2). The presence of the phosphonic acid moiety corresponds to the absorption bands at 1019 and $948 \mathrm{~cm}^{-1}$ for $(\mathrm{P}-\mathrm{OH})$ and $(\mathrm{P}-\mathrm{O})$ groups, respectively. Also the absorption bands of $(\mathrm{P}=\mathrm{O})$ and $(\mathrm{Si}-\mathrm{O}-\mathrm{Si})$ have overlapped and appeared at $\sim 1030-1173 \mathrm{~cm}^{-1} .{ }^{[2,3]}$ The very broad band extending from $3300 \mathrm{~cm}^{-1}$ to as low as $2500 \mathrm{~cm}^{-1}$ is likely to be due to the absorption characteristic of the phosphonic acid hydroxyl group $(\mathrm{OH})$. The N-H stretching band at $3500 \mathrm{~cm}^{-1}$ decreased, which reflected the successful amine conversion by the Mannich reaction.

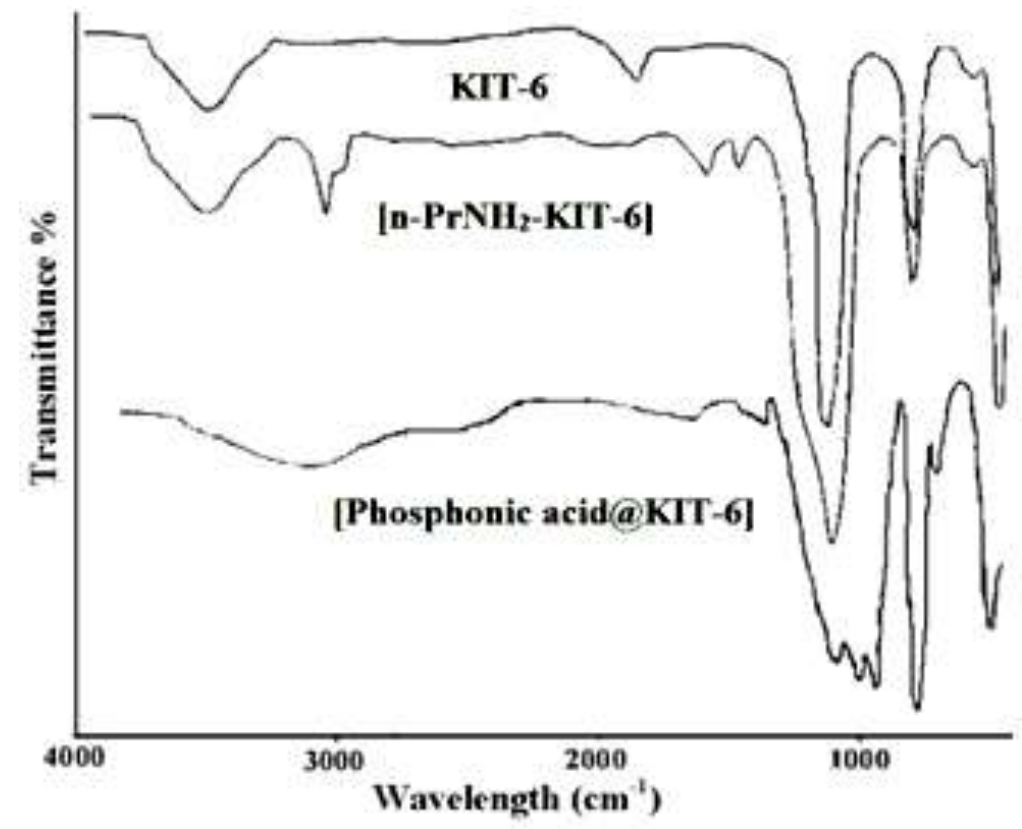

Figure S2. FTIR Spectrum of MSNs 


\section{- Thermal gravimetric analysis (TGA)}

Figure S3 shows the TGA profiles obtained for KIT-6 and [Phosphonic acid@KIT-6]. The loading of aminopropyl functional onto KIT-6 was conclude about $14 \%$ (2.4 mmol.g${ }^{1}$ ) which confirmed by acid-base back titration. Also in the case of [Phosphonic acid@KIT-6], the TGA analysis has showed the weight loss about 44\% in the second step, which is attributed to the removal of organo-phosphonic acid. Therefore, the loading of the organo-phosphorous moiety onto the surface of KIT- 6 is about $3.5 \mathrm{mmol} . \mathrm{g}^{-1}$. Applying acid-base titration method, ${ }^{[3]}$ the exact amount of phosphonic acid groups and ion-exchange capacity of [Phosphonic acid@KIT-6] was 3.6 mmol.g ${ }^{-1}$.

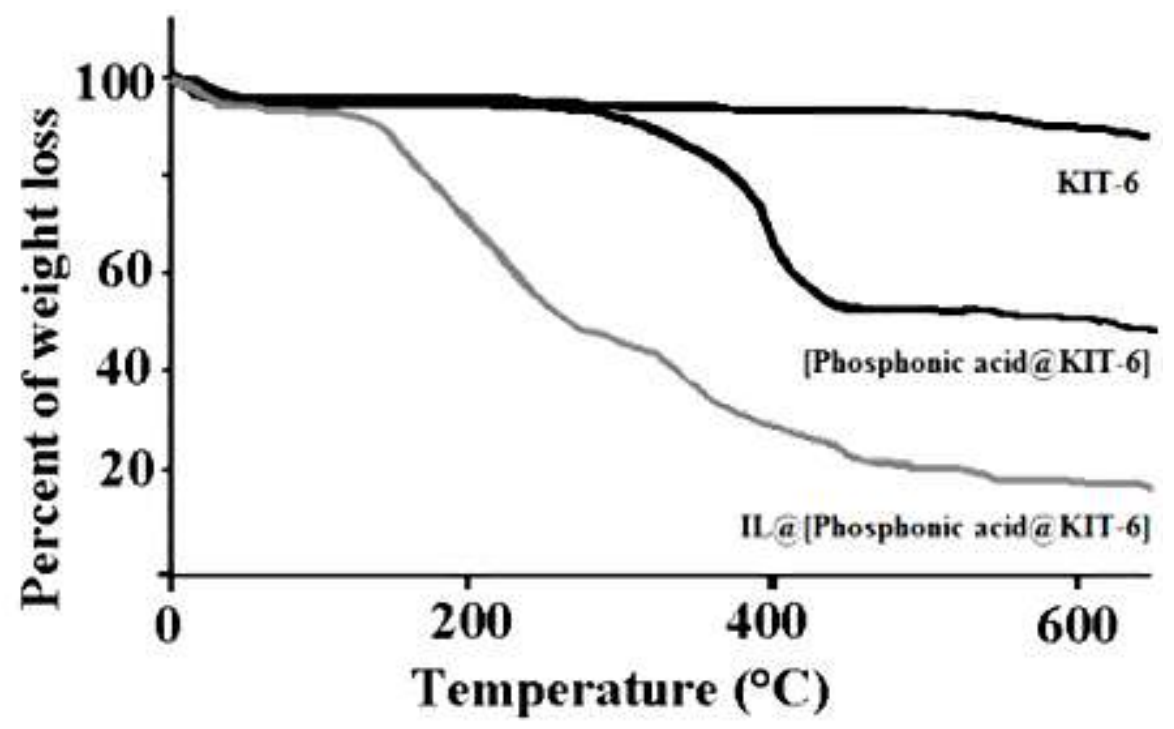

Figure S3. TGA diagrams of MSNs 


\section{- $\quad N_{2}$ sorption isotherm experiment}

The $\mathrm{N}_{2}$ sorption isotherm experiment has revealed a high pore volume and surface area for the synthesized KIT-6. The calculated multiple-point BET (Barrett-Joyner-Halenda) surface area, Barrett-Joyner-Halenda (BJH) average pore size distributions and primary pore volume for KIT-6 were $821.41 \mathrm{~m}^{2} \cdot \mathrm{g}^{-1}, 7.2 \mathrm{~nm}$, and $1.51 \mathrm{~cm}^{3} \cdot \mathrm{g}^{-1}$, respectively (Figure S4 and Table S1). In the case of IL@ [Phosphonic acid@KIT-6], the decrease in the BET surface area and pore volume and the loss of the capillary condensationevaporation $\mathrm{N}_{2}$ isotherm demonstrate that the filling of ionic liquid into the mesochannels of [Phosphonic acid@KIT-6] has successfully been occurred.

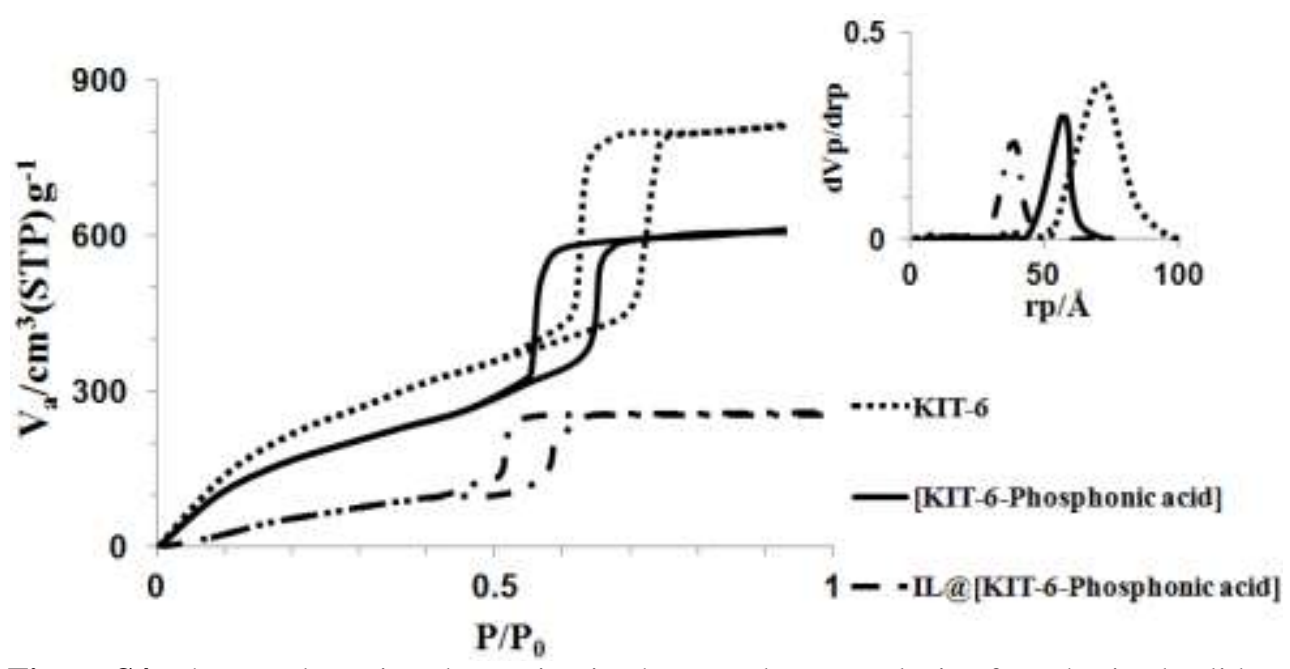

Figure S4. The $\mathrm{N}_{2}$ adsorption-desorption isotherm and $\mathrm{BJH}$ analysis of synthesized solids Table S1. Typical results obtained from BET and BJH before and after functionalization

\begin{tabular}{cccc}
\hline Mesoporous material & $\begin{array}{c}\mathrm{S}_{\mathrm{BET}} \\
\left(\mathrm{m}^{2} \mathrm{~g}^{-1}\right)\end{array}$ & $\begin{array}{c}\mathrm{V}_{\text {total }} \\
\left(\mathrm{cm}^{3} \mathrm{~g}^{-1}\right)\end{array}$ & $\begin{array}{c}\text { Pore diameter } \\
(\mathrm{nm})\end{array}$ \\
\hline KIT-6 & 821.41 & 1.51 & 7.2 \\
[Phosphonic acid@KIT-6] & 575.6 & 0.485 & 5.7 \\
IL@[Phosphonic acid@KIT-6] & 255.3 & 0.108 & 3.9 \\
\hline
\end{tabular}


${ }^{1} \mathrm{H}$ NMR and ${ }^{13} \mathrm{C}$ NMR data of the products

2-(4-Methoxy-5H-chromeno[2,3-d]pyrimidin-2-yl)phenol (5a): Colorless needle crystals; mp 198-200 ${ }^{\circ} \mathrm{C}$ (lit. mp $\left.200{ }^{\circ} \mathrm{C}\right){ }^{[4]} ;{ }^{1} \mathrm{H}$ NMR $\left(\mathrm{CDCl}_{3}, 500 \mathrm{MHz}\right): \delta_{\mathrm{H}}=3.97$ (s, 2H, $\left.\mathrm{CH}_{2}\right), 4.18\left(\mathrm{~s}, 3 \mathrm{H}, \mathrm{OCH}_{3}\right), 6.97(\mathrm{dt}, J=7.5,1.0 \mathrm{~Hz}, 1 \mathrm{H}), 7.04(\mathrm{dd}, J=8.0 \mathrm{~Hz}, 1 \mathrm{H})$, $7.15(\mathrm{dt}, J=7.5 \mathrm{~Hz}, 1.0 \mathrm{~Hz}, 1 \mathrm{H}), 7.19(\mathrm{~d}, J=7.5 \mathrm{~Hz}, 1 \mathrm{H}), 7.23-7.29(\mathrm{~m}, 2 \mathrm{H}), 7.41(\mathrm{dt}, J=$ 8.0, 1.6 Hz, 1H), $8.46(\mathrm{dd}, J=8.0,1.6 \mathrm{~Hz}, 1 \mathrm{H}), 12.78(\mathrm{br} \mathrm{s}, 1 \mathrm{H}, \mathrm{OH}) ;{ }^{13} \mathrm{C} \mathrm{NMR}\left(\mathrm{CDCl}_{3}\right.$, $125 \mathrm{MHz}): \delta_{\mathrm{C}}=21.30,54.22,94.41,116.84,117.31,117.86,118.43,124.16,127.76$, $128.70,128.75,132.62,136.81,149.88,159.78,161.70,167.14$; Anal. Calcd for $\mathrm{C}_{18} \mathrm{H}_{14} \mathrm{~N}_{2} \mathrm{O}_{3}:$ C, 70.58; H, 4.61; N, 9.15; Found: C, 70.72; H, 4.90; N, 9.38.

2-(4,9-dimethoxy-5H-chromeno[2,3-d]pyrimidin-2-yl)phenol (5b): Colorless needle crystals; mp 200-202 ${ }^{\circ} \mathrm{C}$ (lit. mp $205{ }^{\circ} \mathrm{C}$ ) ${ }^{[4]} ;{ }^{1} \mathrm{H}$ NMR $\left(\mathrm{CDCl}_{3}, 400 \mathrm{MHz}\right): \delta_{\mathrm{H}}=3.94$ (s, $\left.2 \mathrm{H}, \mathrm{CH}_{2}\right), 3.95\left(\mathrm{~s}, 3 \mathrm{H}, \mathrm{OCH}_{3}\right), 4.18\left(\mathrm{~s}, 3 \mathrm{H}, \mathrm{OCH}_{3}\right), 6.80(\mathrm{~d}, J=7.6 \mathrm{~Hz}, 1 \mathrm{H}), 6.85(\mathrm{~d}, J=$ $7.6 \mathrm{~Hz}, 1 \mathrm{H}), 6.94(\mathrm{dt}, J=8.0,0.8 \mathrm{~Hz}, 1 \mathrm{H}), 7.01(\mathrm{dd}, J=8.0,0.8 \mathrm{~Hz}, 1 \mathrm{H}), 7.06(\mathrm{t}, J=7.6$ $\mathrm{Hz}, 1 \mathrm{H}), 7.39(\mathrm{dt}, J=8.0,1.6 \mathrm{~Hz}, 1 \mathrm{H}), 8.48(\mathrm{dd}, J=8.0,1.6 \mathrm{~Hz}, 1 \mathrm{H}), 12.77(\mathrm{br} \mathrm{s}, 1 \mathrm{H}$, $\mathrm{OH}) ;{ }^{13} \mathrm{C} \mathrm{NMR}\left(\mathrm{CDCl}_{3}, 125 \mathrm{MHz}\right): \delta_{\mathrm{C}}=21.49,54.23,55.58,94.26,110.06,117.27$, $118.02,118.34,119.47,120.02,123.85,128.97,132.53,139.46,147.76,159.66,161.75$, 162.66, 167.37; Anal. Calcd for $\mathrm{C}_{19} \mathrm{H}_{16} \mathrm{~N}_{2} \mathrm{O}_{4}$ : C, 67.85; H, 4.79; N, 8.33; Found: C, $68.15 ; \mathrm{H}, 4.99 ; \mathrm{N}, 8.12$.

- 2-(7-bromo-4-methoxy-5H-chromeno[2,3-d]pyrimidin-2-yl)phenol (5c): Colorless needle crystals; mp 240-242 ${ }^{\circ} \mathrm{C}$ (lit. mp $\left.245{ }^{\circ} \mathrm{C}\right)^{[4]} ;{ }^{1} \mathrm{H}$ NMR $\left(\mathrm{CDCl}_{3}, 500 \mathrm{MHz}\right): \delta_{\mathrm{H}}=$ 3.92(s, $\left.2 \mathrm{H}, \mathrm{CH}_{2}\right), 4.18\left(\mathrm{~s}, 3 \mathrm{H}, \mathrm{OCH}_{3}\right), 6.95(\mathrm{dt}, J=8.0,0.8 \mathrm{~Hz}, 1 \mathrm{H}), 7.02(\mathrm{~d}, J=8.0 \mathrm{~Hz}$, 1H), $7.05(\mathrm{~d}, J=8.0 \mathrm{~Hz}, 1 \mathrm{H}), 7.36-7.41(\mathrm{~m}, 3 \mathrm{H}), 8.42(\mathrm{dd}, J=8.0,1.0 \mathrm{~Hz}, 1 \mathrm{H}), 12.70(\mathrm{br}$ s, $1 \mathrm{H}, \mathrm{OH}) ;{ }^{13} \mathrm{CNMR}\left(\mathrm{CDCl}_{3}, 125 \mathrm{MHz}\right): \delta_{\mathrm{C}}=21.69,54.97,94.55,117.00,117.90$, 
$118.34,119.05,119.11,121.30,129.19,131.45,132.03,133.37,134.01,149.75,160.23$, 161.85, 167.73; Anal. Calcd for $\mathrm{C}_{18} \mathrm{H}_{13} \mathrm{BrN}_{2} \mathrm{O}_{3}$ : C, 56.12; H, 3.40; N, 7.27; Found: C, $56.25 ; \mathrm{H}, 3.18 ; \mathrm{N}, 7.50$.

- 2-(4-ethoxy-5H-chromeno[2,3-d]pyrimidin-2-yl)phenol (5d): Colorless needle crystals; mp 197-199 ${ }^{\circ} \mathrm{C}\left(\text { lit. mp } 200{ }^{\circ} \mathrm{C}\right)^{[4]} ;{ }^{1} \mathrm{H}$ NMR $\left(\mathrm{CDCl}_{3}, 400 \mathrm{MHz}\right): \delta_{\mathrm{H}}=1.50(\mathrm{t}, J=$ $\left.7.0 \mathrm{~Hz}, 3 \mathrm{H}, \mathrm{CH}_{3}\right), 3.93\left(\mathrm{~s}, 2 \mathrm{H}, \mathrm{CH}_{2}\right) 4.57\left(\mathrm{q}, J=7.0 \mathrm{~Hz}, 2 \mathrm{H}, \mathrm{OCH}_{2}\right), 6.94(\mathrm{t}, J=7.5,1.0$ $\mathrm{Hz}, 1 \mathrm{H}), 7.01(\mathrm{~d}, J=8.0 \mathrm{~Hz}, 1 \mathrm{H}), 7.11(\mathrm{t}, J=8.0 \mathrm{~Hz}, 1 \mathrm{H}), 7.15(\mathrm{~d}, J=8.0 \mathrm{~Hz}, 1 \mathrm{H}), 7.20-$ $7.26(\mathrm{~m}, 2 \mathrm{H}), 7.38(\mathrm{t}, J=8.0 \mathrm{~Hz}, 1 \mathrm{H}), 8.40(\mathrm{~d}, J=8.0 \mathrm{~Hz}, 1 \mathrm{H}), 12.79$ (br s, 1H, OH); ${ }^{13} \mathrm{CNMR}\left(\mathrm{CDCl}_{3}, 125 \mathrm{MHz}\right): \delta_{\mathrm{C}}=9.65,21.38,64.09,94.39,116.23,117.50,117.87$, $118.48,118.73,122.45,123.96,127.59,128.57,128.71,133.07,159.68,161.53,161.80$ 166.95; Anal. Calcd for $\mathrm{C}_{19} \mathrm{H}_{16} \mathrm{~N}_{2} \mathrm{O}_{3}$ : C, 71.24; H, 5.03; N, 8.74; Found: C, 71.45; H, $5.20 ; \mathrm{N}, 8.51$.

- 2-(4-Ethoxy-9-methoxy-5H-chromeno[2,3-d]pyrimidin-2-yl)phenol (5e): Colorless needle crystals; mp 198-200 ${ }^{\circ} \mathrm{C}$ (lit. mp $\left.200{ }^{\circ} \mathrm{C}\right)^{[4]},{ }^{1} \mathrm{H}$ NMR $\left(\mathrm{CDCl}_{3}, 400 \mathrm{MHz}\right): \delta_{\mathrm{H}}=1.52$ (t, $\left.J=9.0 \mathrm{~Hz}, 3 \mathrm{H}, \mathrm{CH}_{3}\right), 3.94\left(\mathrm{~s}, 2 \mathrm{H}, \mathrm{CH}_{2}\right), 3.95\left(\mathrm{~s}, 3 \mathrm{H}, \mathrm{OCH}_{3}\right), 4.59$ (q, $J=9.0 \mathrm{~Hz}, 2 \mathrm{H}$, $\left.\mathrm{OCH}_{2}\right), 6.80(\mathrm{~d}, J=8.0 \mathrm{~Hz}, 1 \mathrm{H}), 6.85(\mathrm{~d}, J=8.0 \mathrm{~Hz}, 1 \mathrm{H}), 6.94(\mathrm{dt}, J=7.8,1.2 \mathrm{~Hz}, 1 \mathrm{H})$, $7.02(\mathrm{dd}, J=7.8,1.2 \mathrm{~Hz}, 1 \mathrm{H}), 7.06(\mathrm{t}, J=8.0 \mathrm{~Hz}, 1 \mathrm{H}), 7.38(\mathrm{dt}, J=7.8,1.6 \mathrm{~Hz}, 1 \mathrm{H}), 8.46$ (dd, $J=7.8,1.6 \mathrm{~Hz}, 1 \mathrm{H}), 12.81$ (br s, $1 \mathrm{H}, \mathrm{OH}) ;{ }^{13} \mathrm{C} \mathrm{NMR}\left(\mathrm{CDCl}_{3}, 125 \mathrm{MHz}\right): \delta_{\mathrm{C}}=14.41$, $21.73,59.03,63.64,94.71,110.54,117.73,118.54,118.82,120.01,120.51,124.82$, $129.60,132.66,140.06,148.42,159.21,160.27,162.06,167.29$; Anal. Calcd for $\mathrm{C}_{20} \mathrm{H}_{18} \mathrm{~N}_{2} \mathrm{O}_{4}$ : C, 68.56; H, 5.18; N, 8.00; Found: C, 68.70; H, 5.32; N, 7.78.

- 2-(7-Bromo-4-ethoxy-5H-chromeno[2,3-d]pyrimidin-2-yl)phenol (5f): Colorless needle crystals; mp 216-219 ${ }^{\circ} \mathrm{C}$ (lit. mp $\left.220{ }^{\circ} \mathrm{C}\right)^{[4]},{ }^{1} \mathrm{H}$ NMR $\left(\mathrm{CDCl}_{3}, 500 \mathrm{MHz}\right): \delta_{\mathrm{H}}=1.52(\mathrm{t}, J=$ 
$\left.6.5 \mathrm{~Hz}, 3 \mathrm{H}, \mathrm{CH}_{3}\right), 3.90\left(\mathrm{~s}, 2 \mathrm{H}, \mathrm{CH}_{2}\right), 4.58$ (q, $\left.2 \mathrm{H}, J=6.5 \mathrm{~Hz}, \mathrm{OCH}_{2}\right), 6.93(\mathrm{t}, J=8.0 \mathrm{~Hz}$, $1 \mathrm{H}), 7.01(\mathrm{~d}, J=7.5 \mathrm{~Hz}, 1 \mathrm{H}), 7.04(\mathrm{~d}, J=8.0 \mathrm{~Hz}, 1 \mathrm{H}), 7.35-7.40(\mathrm{~m}, 3 \mathrm{H}), 8.39(\mathrm{~d}, J=8.0$ $\mathrm{Hz}, 1 \mathrm{H}), 12.70$ (br s, $1 \mathrm{H}, \mathrm{OH}) ;{ }^{13} \mathrm{C} \mathrm{NMR}\left(\mathrm{CDCl}_{3}, 125 \mathrm{MHz}\right): \delta_{\mathrm{C}}=14.49,21.67,63.90$, $94.24,117.09,117.87,118.71,118.99,119.08,120.01,122.41,126.52,129.28,131.30$, 131.93, 133.37, 149.50, 160.48, 162.58; Anal. Calcd for $\mathrm{C}_{19} \mathrm{H}_{15} \mathrm{BrN}_{2} \mathrm{O}_{3}: \mathrm{C}, 57.16 ; \mathrm{H}$, 3.79; N, 7.02; Found: C, 57.36; H, 3.39; N, 7.36.

2-(4-Propoxy-5H-chromeno[2,3-d]pyrimidin-2-yl)phenol(5g): Colorless needle crystals; mp 147-149 ${ }^{\circ} \mathrm{C}$ (lit. mp $\left.150{ }^{\circ} \mathrm{C}\right)^{[4]},{ }^{1} \mathrm{H}$ NMR $\left(\mathrm{CDCl}_{3}, 400 \mathrm{MHz}\right): \delta_{\mathrm{H}}=1.11(\mathrm{t}, J=7.4 \mathrm{~Hz}$, $\left.3 \mathrm{H}, \mathrm{CH}_{3}\right), 1.92\left(\mathrm{~m}, 2 \mathrm{H}, \mathrm{CH}_{2}\right), 3.95\left(\mathrm{~s}, 2 \mathrm{H}, \mathrm{CH}_{2}\right), 4.48\left(\mathrm{q}, J=6.8 \mathrm{~Hz}, 2 \mathrm{H}, \mathrm{OCH}_{2}\right), 6.95$ $(\mathrm{dt}, J=8.0,1.0 \mathrm{~Hz}, 1 \mathrm{H}), 7.03(\mathrm{dd}, J=8.0,0.8 \mathrm{~Hz}, 1 \mathrm{H}), 7.13(\mathrm{dt}, J=8.0,0.8 \mathrm{~Hz}, 1 \mathrm{H})$, $7.17(\mathrm{dd}, J=8.0,1.0 \mathrm{~Hz}, 1 \mathrm{H}), 7.22-7.27(\mathrm{~m}, 2 \mathrm{H}), 7.38(\mathrm{dt}, J=8.0,1.4 \mathrm{~Hz}, 1 \mathrm{H}), 8.42(\mathrm{dd}$, $J=8.0,1.4 \mathrm{~Hz}, 1 \mathrm{H}), 12.83($ br s, $1 \mathrm{H}, \mathrm{OH}) ;{ }^{13} \mathrm{C} \mathrm{NMR}\left(\mathrm{CDCl}_{3}, 125 \mathrm{MHz}\right): \delta_{\mathrm{C}}=10.48$, $21.80,22.19,69.45,94.91,117.34,117.76,118.46,118.91,119.07,124.63,128.25$, 129.20, 133.05, 151.37, 160.22, 162.18, 163.25,167.34; Anal. Calcd for $\mathrm{C}_{20} \mathrm{H}_{18} \mathrm{~N}_{2} \mathrm{O}_{3}$ : C, 71.84; H, 5.43; N, 8.38; Found: C, 72.05; H, 5.62; N, 8.12.

2-(4-Butoxy-5H-chromeno[2,3-d]pyrimidin-2-yl)phenol (5h): Colorless needle crystals; mp 142-144 ${ }^{\circ} \mathrm{C}$ (lit. mp $\left.145^{\circ} \mathrm{C}\right)^{[4]} ;{ }^{1} \mathrm{H}$ NMR $\left(\mathrm{CDCl}_{3}, 400 \mathrm{MHz}\right): \delta_{\mathrm{H}}=1.04$ (t, $3 \mathrm{H}, J=9.5$ $\left.\mathrm{Hz}, \mathrm{CH}_{3}\right), 1.51-1.61\left(\mathrm{~m}, 2 \mathrm{H}, \mathrm{CH}_{2}\right), 1.84-1.91\left(\mathrm{~m}, 2 \mathrm{H}, \mathrm{CH}_{2}\right), 3.95\left(\mathrm{~s}, 2 \mathrm{H}, \mathrm{CH}_{2}\right), 4.53$ (t, $J$ $\left.=6.4 \mathrm{~Hz}, 2 \mathrm{H}, \mathrm{OCH}_{2}\right), 6.95(\mathrm{dt}, J=8.0,1.2 \mathrm{~Hz}, 1 \mathrm{H}), 7.02(\mathrm{dd}, J=8.0,0.8 \mathrm{~Hz}, 1 \mathrm{H}), 7.14$ $(\mathrm{dt}, J=8.0,0.8 \mathrm{~Hz}, 1 \mathrm{H}), 7.17(\mathrm{~d}, J=8.0 \mathrm{~Hz}, 1 \mathrm{H}), 7.23-7.27(\mathrm{~m}, 2 \mathrm{H}), 7.39(\mathrm{dt}, J=8.0$, $1.6 \mathrm{~Hz}, 1 \mathrm{H}), 8.43(\mathrm{dd}, J=8.0,1.6 \mathrm{~Hz}, 1 \mathrm{H}), 12.84(\mathrm{br} \mathrm{s}, 1 \mathrm{H}, \mathrm{OH}) ;{ }^{13} \mathrm{C} \mathrm{NMR}\left(\mathrm{CDCl}_{3}, 125\right.$ $\mathrm{MHz}): \delta_{\mathrm{C}}=13.57,19.01,21.43,30.41,56.04,67.87,94.50,110.42,117.31,118.06$, $118.48,118.65,124.21,127.80,128.44,128.78,132.65,149.93,159.79,161.70,166.45$, 
168.73; Anal. Calcd for $\mathrm{C}_{21} \mathrm{H}_{20} \mathrm{~N}_{2} \mathrm{O}_{3}$ : C, 72.40; H, 5.79; N, 8.04; Found: C, 72.61; H, $5.44 ; \mathrm{N}, 8.27$.

2-(4-Butoxy-9-methoxy-5H-chromeno[2,3-d]pyrimidin-2-yl)phenol (5i): Colorless needle crystals; mp 151-153 ${ }^{\circ} \mathrm{C}\left(\text { lit. } \mathrm{mp} 156{ }^{\circ} \mathrm{C}\right)^{[4]}$; ${ }^{1} \mathrm{H}$ NMR $\left(\mathrm{CDCl}_{3}, 400 \mathrm{MHz}\right): \delta_{\mathrm{H}}=1.04$ (t, $\left.3 \mathrm{H}, J=7.5 \mathrm{~Hz}, \mathrm{CH}_{3}\right), 1.51-1.60\left(\mathrm{~m}, 2 \mathrm{H}, \mathrm{CH}_{2}\right), 1.84-1.91\left(\mathrm{~m}, 2 \mathrm{H}, \mathrm{CH}_{2}\right), 3.94(\mathrm{~s}, 2 \mathrm{H}$, $\left.\mathrm{CH}_{2}\right), 3.95\left(\mathrm{~s}, 3 \mathrm{H}, \mathrm{OCH}_{3}\right), 4.53\left(\mathrm{t}, 2 \mathrm{H}, J=6.4 \mathrm{~Hz} \mathrm{OCH}_{2}\right), 6.81(\mathrm{~d}, J=8.0 \mathrm{~Hz}, 1 \mathrm{H}), 6.85$ $(\mathrm{d}, J=8.0 \mathrm{~Hz}, 1 \mathrm{H}, 1 \mathrm{CH}), 6.94(\mathrm{t}, J=7.8, \mathrm{~Hz}, 1 \mathrm{H}), 7.01(\mathrm{~d}, J=7.8 \mathrm{~Hz}, 1 \mathrm{H}), 7.08(\mathrm{t}, J=$ $7.8 \mathrm{~Hz}, 1 \mathrm{H}), 7.38(\mathrm{dt}, J=7.8,1.6 \mathrm{~Hz}, 1 \mathrm{H}), 8.47(\mathrm{dd}, J=8.0,1.6 \mathrm{~Hz}, 1 \mathrm{H}), 12.82(\mathrm{br} \mathrm{s}$, $1 \mathrm{H}, \mathrm{OH}) ;{ }^{13} \mathrm{C} \mathrm{NMR}\left(\mathrm{CDCl}_{3}, 125 \mathrm{MHz}\right): \delta_{\mathrm{C}}=13.92,19.29,22.01,30.79,56.10,67.59$, $94.77,110.49,117.80,118.56,118.91,120.09,120.66,124.48,129.50,132.09,140.11$, 148.39, 160.20, 162.24, 163.12, 167.68; Anal. Calcd for $\mathrm{C}_{22} \mathrm{H}_{22} \mathrm{~N}_{2} \mathrm{O}_{4}$ : C, 69.83; H, 5.86; N, 7.40; Found: C, 69.51; H, 6.01; N, 7.59.

- 2-(7-Bromo-4-butoxy-5H-chromeno[2,3-d]pyrimidin-2-yl)phenol (5j): Colorless needle crystals; mp 215-217 ${ }^{\circ} \mathrm{C}$ (lit. mp $220{ }^{\circ} \mathrm{C}$ ) ${ }^{[4]}$; ${ }^{1} \mathrm{H}$ NMR $\left(\mathrm{CDCl}_{3}, 500 \mathrm{MHz}\right): \delta_{\mathrm{H}}=1.04(\mathrm{t}, 3 \mathrm{H}$, $\left.J=7.40 \mathrm{~Hz}, \mathrm{CH}_{3}\right), 1.51-1.57\left(\mathrm{~m}, 2 \mathrm{H}, \mathrm{CH}_{2}\right), 1.83-1.89(\mathrm{~m}, 2 \mathrm{H}), 3.90\left(\mathrm{~s}, 2 \mathrm{H}, \mathrm{CH}_{2}\right), 4.51(\mathrm{t}$, $\left.2 \mathrm{H}, J=6.5 \mathrm{~Hz}, \mathrm{OCH}_{2}\right), 6.94(\mathrm{dt}, J=7.8 \mathrm{~Hz}, 1.0 \mathrm{~Hz}, 1 \mathrm{H}), 7.01(\mathrm{~d}, J=7.8 \mathrm{~Hz}, 1 \mathrm{H}), 7.04$ (d, $J=8.0 \mathrm{~Hz}, 1 \mathrm{H}), 7.35-7.39(\mathrm{~m}, 3 \mathrm{H}), 8.39(\mathrm{~d}, J=7.8 \mathrm{~Hz}, 1 \mathrm{H}), 12.70(\mathrm{br} \mathrm{s}, 1 \mathrm{H}, \mathrm{OH})$; ${ }^{13} \mathrm{C}$ NMR $\left(\mathrm{CDCl}_{3}, 125 \mathrm{MHz}\right): \delta_{\mathrm{C}}=13.94,19.32,22.01,30.92,67.66,94.31,117.02$, $117.90,118.12,118.99,119.13,121.41,129.28,131.33,132.08,133.27,149.56,159.89$ 162.52, 162.95, 167.67; Anal. Calcd for $\mathrm{C}_{21} \mathrm{H}_{19} \mathrm{BrN}_{2} \mathrm{O}_{3}$ : C, 59.03; H, 4.48; N, 6.56; Found: C, 59.25; H, 4.23; N, 6.80 .

- 2-(4-isobutoxy-5H-chromeno[2,3-d]pyrimidin-2-yl)phenol (5k): Colorless needle crystals; mp 237-239 ${ }^{\circ} \mathrm{C}\left(\right.$ lit. mp $\left.240{ }^{\circ} \mathrm{C}\right){ }^{[4]} ;{ }^{1} \mathrm{H} \mathrm{NMR}\left(\mathrm{CDCl}_{3}, 400 \mathrm{MHz}\right): \delta_{\mathrm{H}}=1.11(\mathrm{~d}, J=$ 
$\left.6.8 \mathrm{~Hz}, 6 \mathrm{H}, 2 \mathrm{CH}_{3}\right), 2.22(\mathrm{~m}, \mathrm{H}, \mathrm{CH}), 3.90\left(\mathrm{~s}, 2 \mathrm{H}, \mathrm{CH}_{2}\right), 4.30\left(\mathrm{~d}, 2 \mathrm{H}, J=6.4 \mathrm{~Hz}, \mathrm{OCH}_{2}\right)$, $6.91(\mathrm{dt}, J=8.0,1.2 \mathrm{~Hz}, 1 \mathrm{H}), 6.98(\mathrm{dd}, J=8.0,0.8 \mathrm{~Hz}, 1 \mathrm{H}), 7.06-7.11(\mathrm{~m}, 2 \mathrm{H}), 7.16-7.21$ (m, 2H), $7.35(\mathrm{dt}, J=8.0,2.0 \mathrm{~Hz}, 1 \mathrm{H}), 8.36(\mathrm{dd}, J=8.0,2.0 \mathrm{~Hz}, 1 \mathrm{H}), 12.80($ br s, $1 \mathrm{H}$, $\mathrm{OH}$ ); Anal. Calcd for $\mathrm{C}_{21} \mathrm{H}_{20} \mathrm{~N}_{2} \mathrm{O}_{3}$ : C, 72.40; H, 5.79; N, 8.04; Found: C, 72.10; H, 5.46; $\mathrm{N}, 8.25$.

- 4-bromo-2-(4-methoxy-5H-chromeno[2,3-d]pyrimidin-2-yl)phenol (5l): Colorless needle crystals; mp 210-212 ${ }^{\circ} \mathrm{C} ;{ }^{1} \mathrm{H}$ NMR $\left(\mathrm{CDCl}_{3}, 400 \mathrm{MHz}\right): \delta_{\mathrm{H}}=3.96\left(\mathrm{~s}, 2 \mathrm{H}, \mathrm{CH}_{2}\right)$, 4.17(s, 3H, $\left.\mathrm{OCH}_{3}\right), 4.22\left(\mathrm{~s}, 3 \mathrm{H}, \mathrm{OCH}_{3}\right), 6.76(\mathrm{~d}, J=8.0,1 \mathrm{H}), 6.92(\mathrm{~d}, J=8.0,1 \mathrm{H}), 7.10-$ $7.41(\mathrm{~m}, 2 \mathrm{H}), 8.45(\mathrm{~s}, 1 \mathrm{H}), 12.83($ br s, $1 \mathrm{H}, \mathrm{OH}) ;{ }^{13} \mathrm{C} \mathrm{NMR}\left(\mathrm{CDCl}_{3}, 125 \mathrm{MHz}\right): \delta_{\mathrm{C}}=$ $21.2,57.0,94.5,114.2,117.8,118.4,119.7,122.4,123.9,127.5,131.2,132.8,133.4$ 149.9, 153.0, 160.2, 161.8, 167.2; Anal. Calcd for $\mathrm{C}_{18} \mathrm{H}_{13} \mathrm{BrN}_{2} \mathrm{O}_{3}: \mathrm{C}, 56.12 ; \mathrm{H}, 3.40 ; \mathrm{N}$, 7.27; Found: C, 56.35; H, 3.79; N, 7.04.

- 4-bromo-2-(7-bromo-4-methoxy-5H-chromeno[2,3-d]pyrimidin-2-yl)phenol (5m): Colorless needle crystals; mp 235-237 ${ }^{\circ} \mathrm{C} ;{ }^{1} \mathrm{H}$ NMR $\left(\mathrm{CDCl}_{3}, 400 \mathrm{MHz}\right): \delta_{\mathrm{H}}=3.97(\mathrm{~s}, 2 \mathrm{H}$, $\left.\mathrm{CH}_{2}\right), 4.18\left(\mathrm{~s}, 3 \mathrm{H}, \mathrm{OCH}_{3}\right), 6.81(\mathrm{~d}, J=8.5 \mathrm{~Hz}, 1 \mathrm{H}), 6.97(\mathrm{~d}, J=8.5,1 \mathrm{H}), 7.26-7.39(\mathrm{~m}$, $8.42(\mathrm{~s}, 1 \mathrm{H}), 12.58($ br s, $1 \mathrm{H}, \mathrm{OH}) ;{ }^{13} \mathrm{C} \mathrm{NMR}\left(\mathrm{CDCl}_{3}, 125 \mathrm{MHz}\right): \delta_{\mathrm{C}}=21.2,56.1,57.5$, $94.9,116.2,117.8,118.4,119.7,121.4,123.9,127.6,132.2,133.8,134.7,149.8,154.7$, 160.2, 161.5, 168.2; Anal. Calcd for $\mathrm{C}_{18} \mathrm{H}_{12} \mathrm{Br}_{2} \mathrm{~N}_{2} \mathrm{O}_{3}$ : C, 46.58; H, 2.61; N, 6.04; Found: C, 46.73; H, 2.53; N, 6.22. 


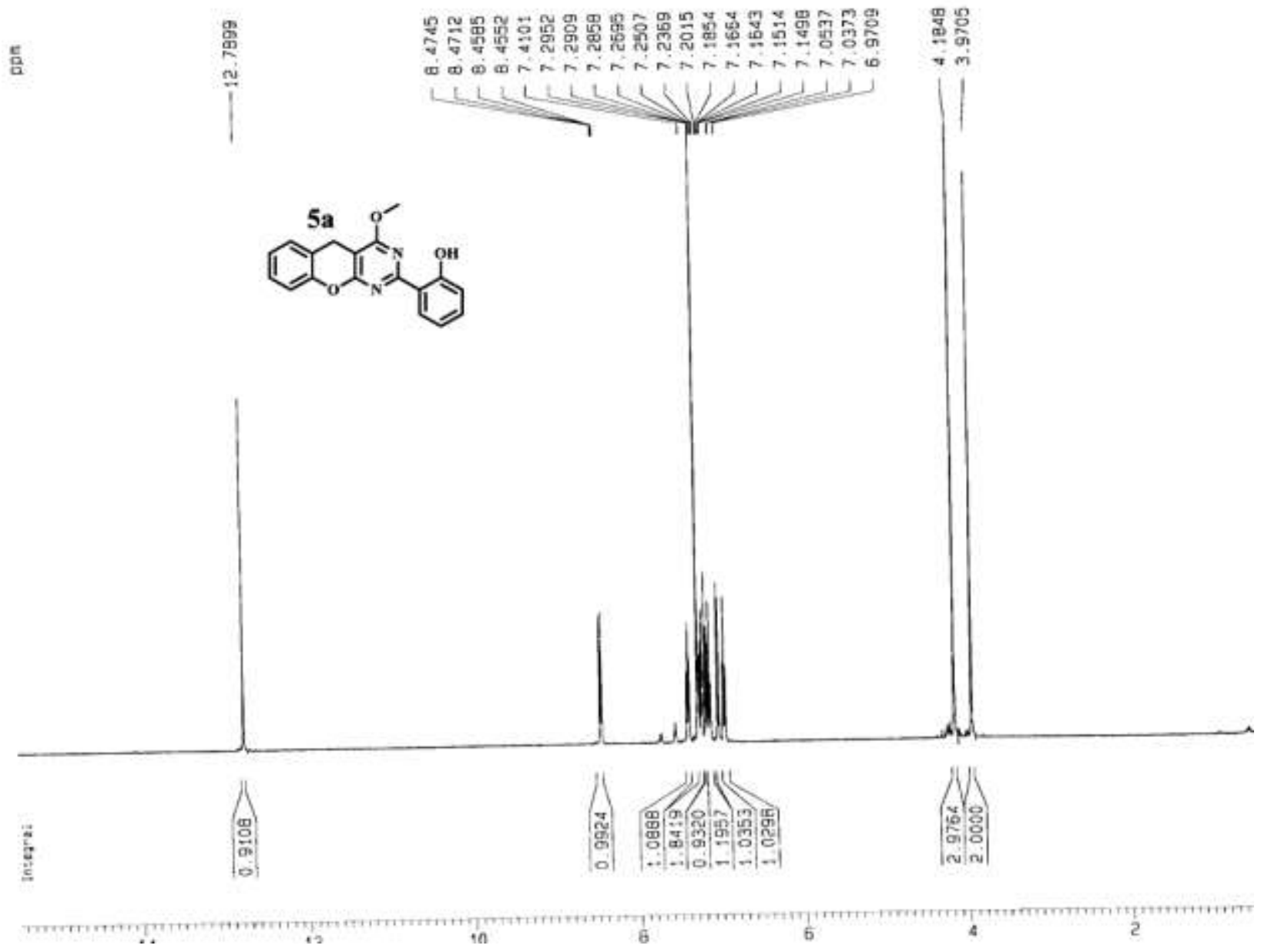



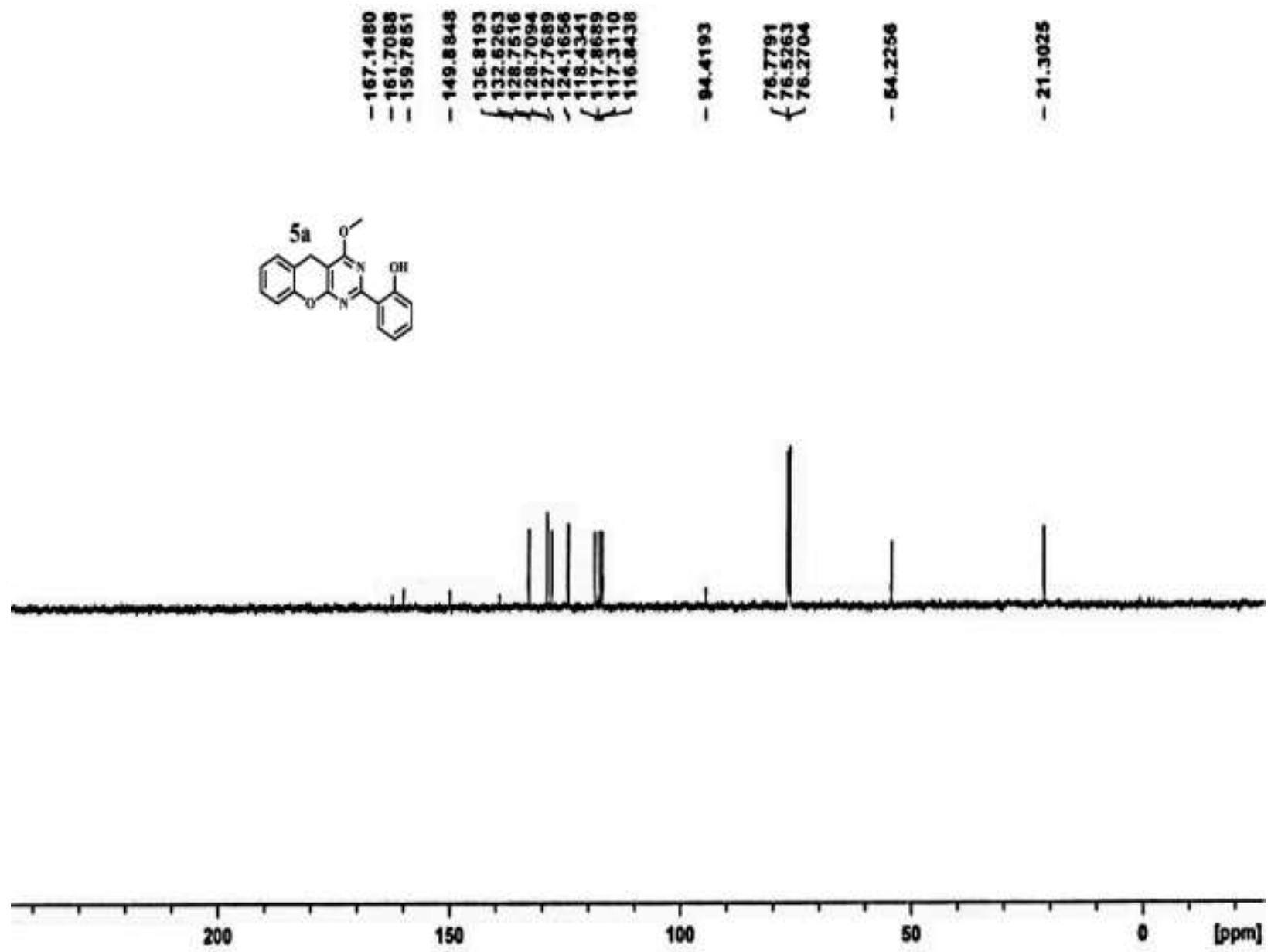


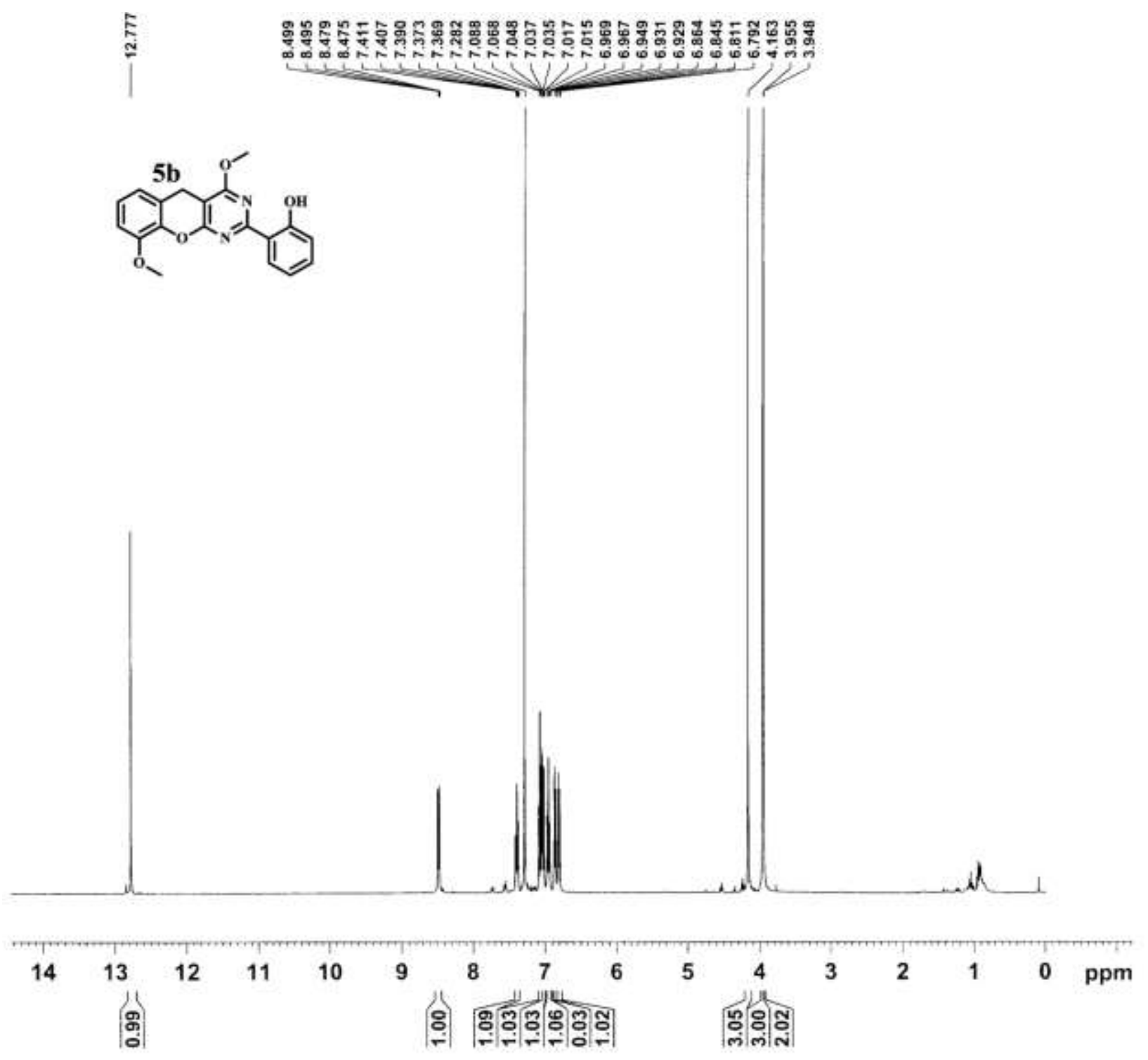



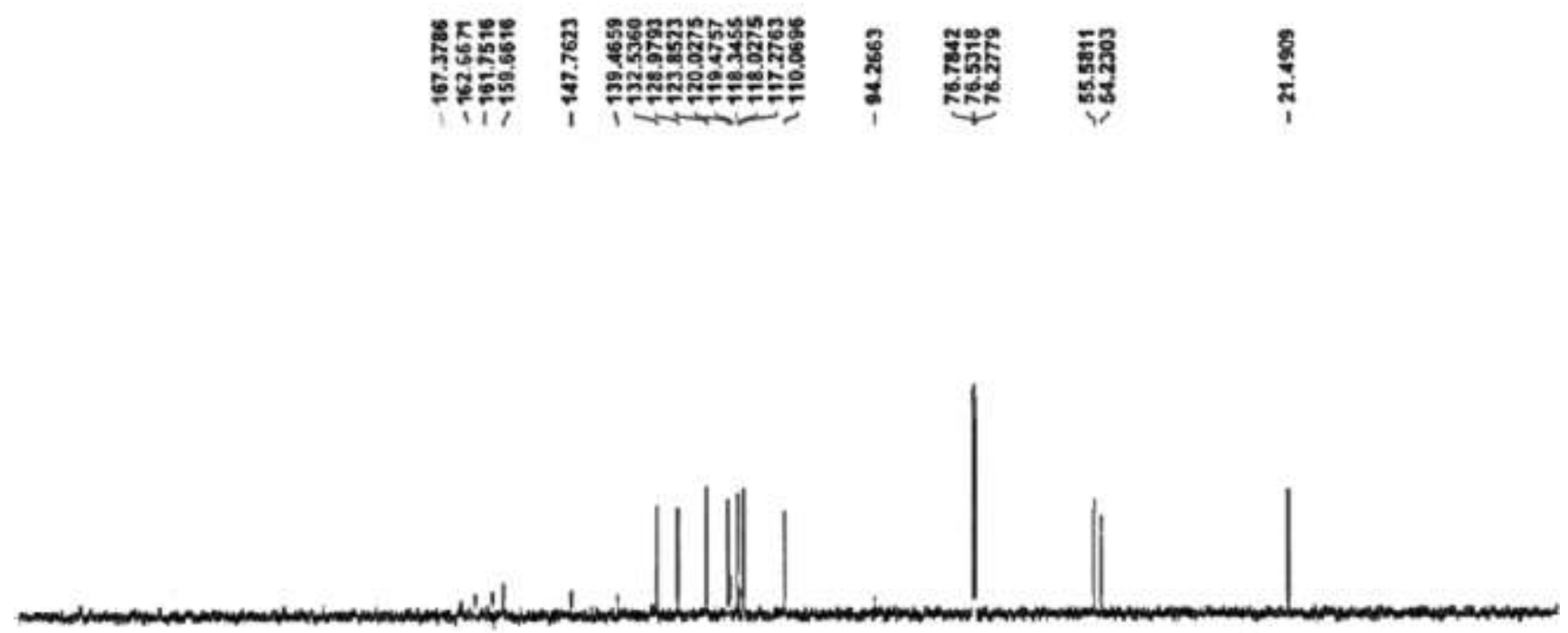

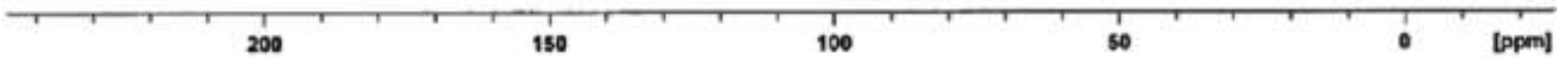




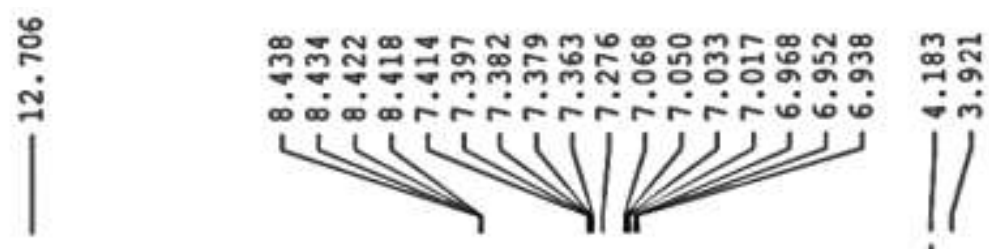<smiles>[CH]Cc1cc(Br)ccc1Oc1nc(-c2ccccc2O)nc(OC)c1C</smiles>
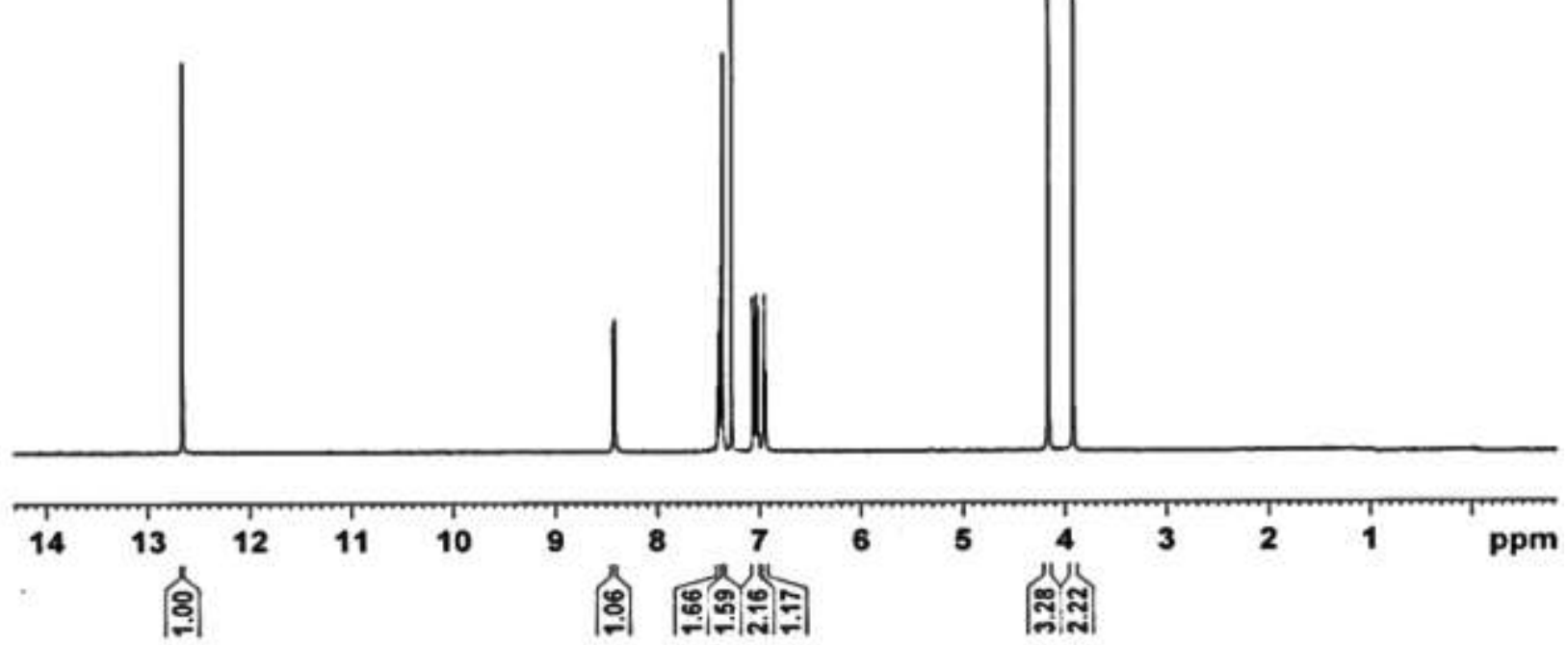


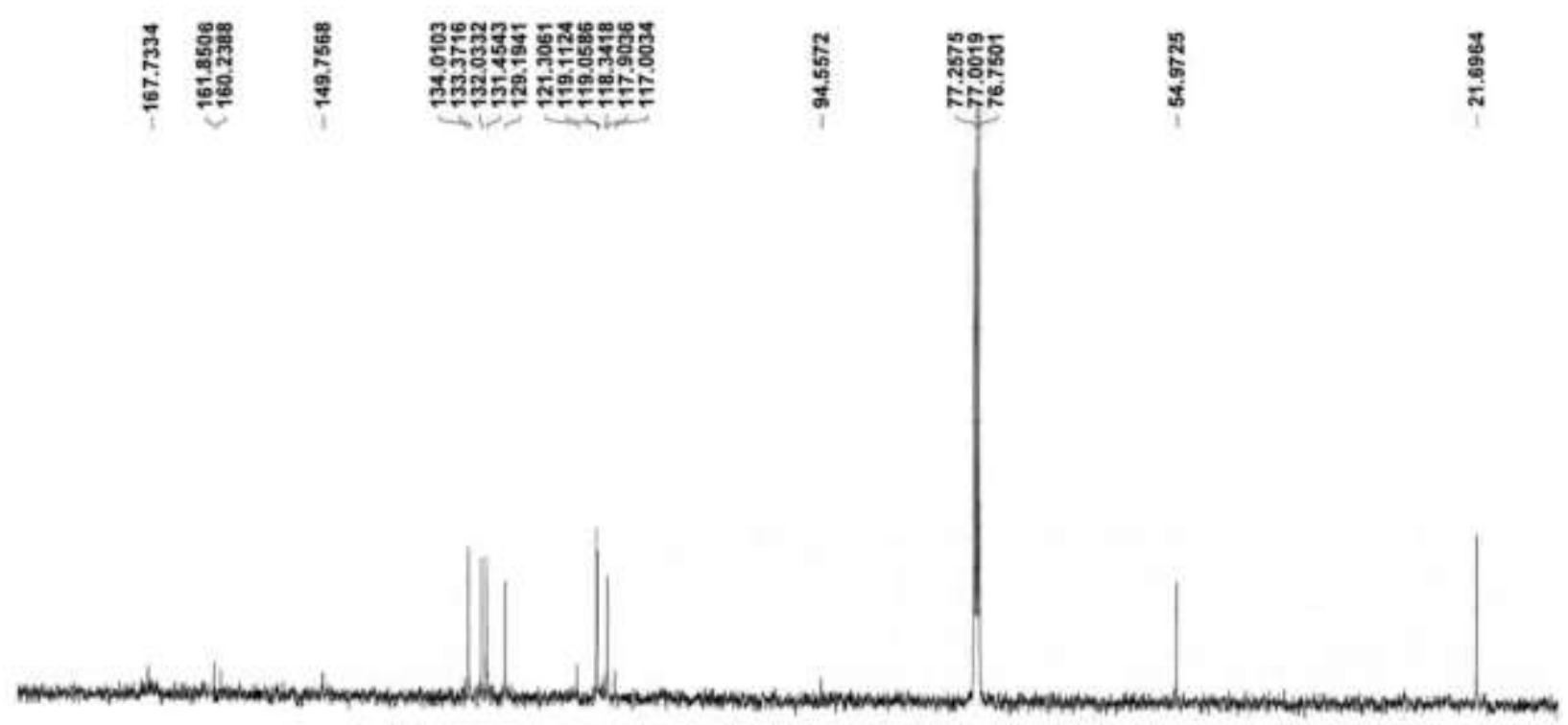

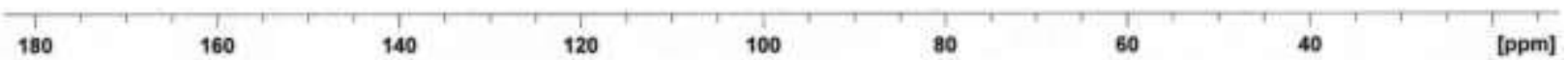



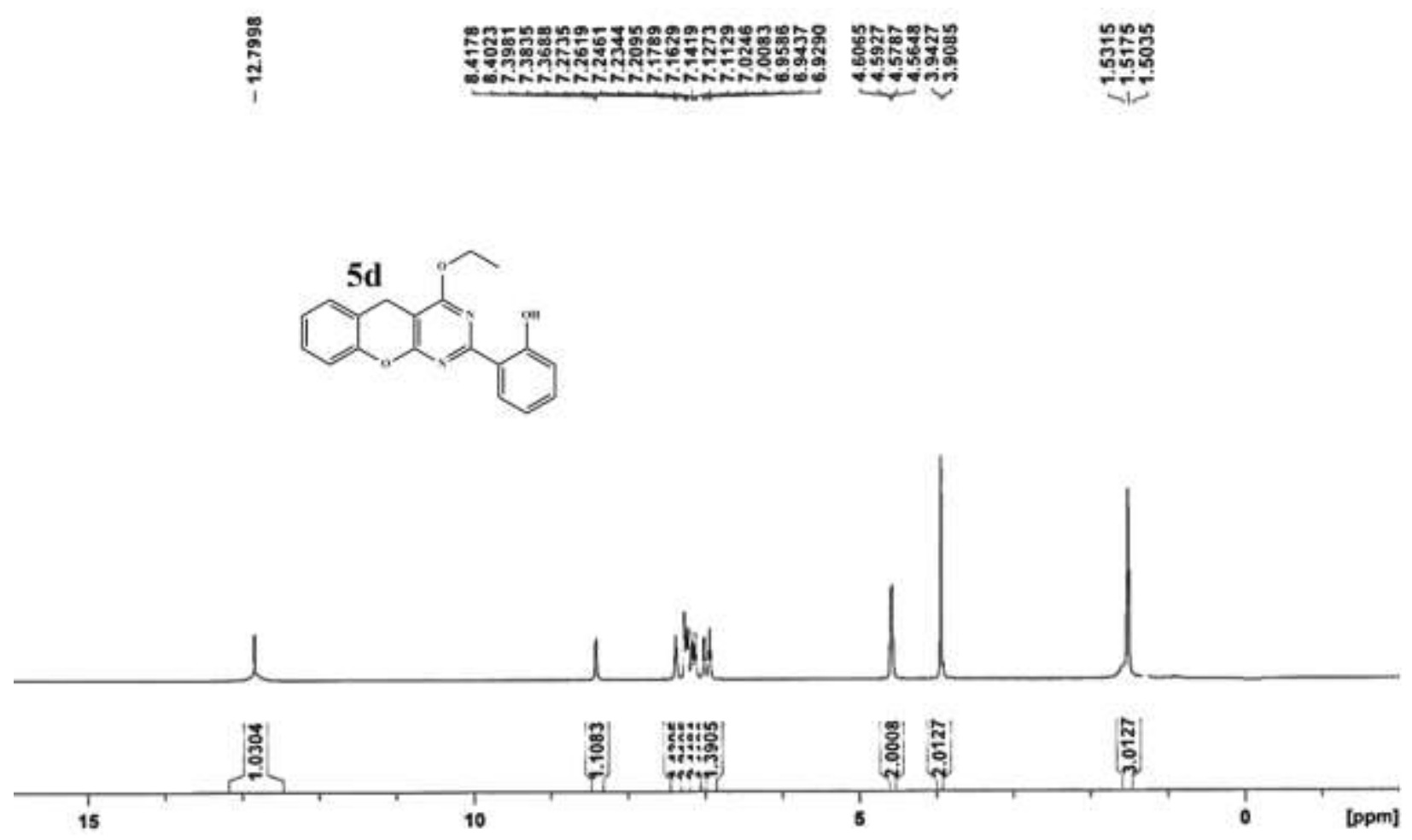

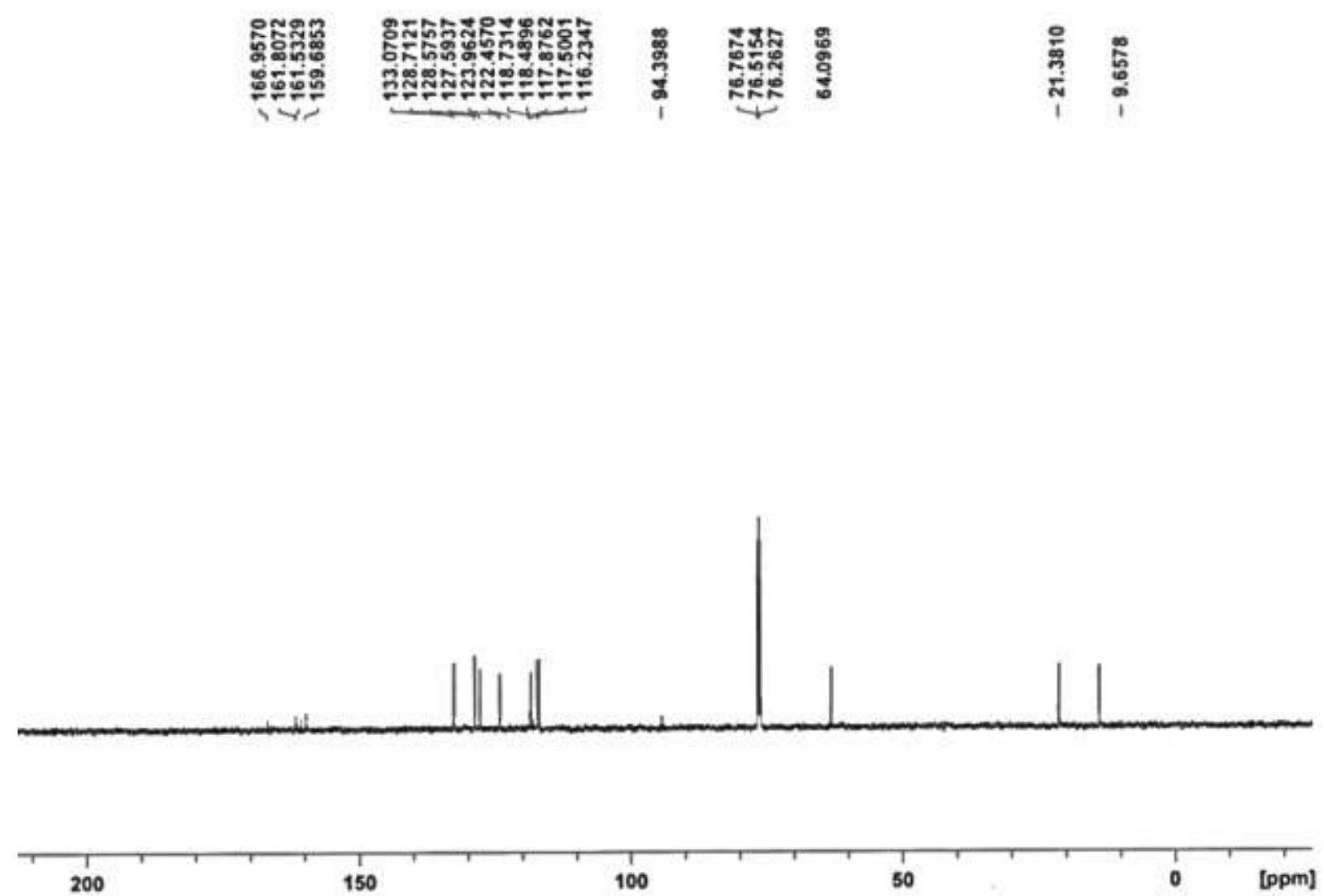


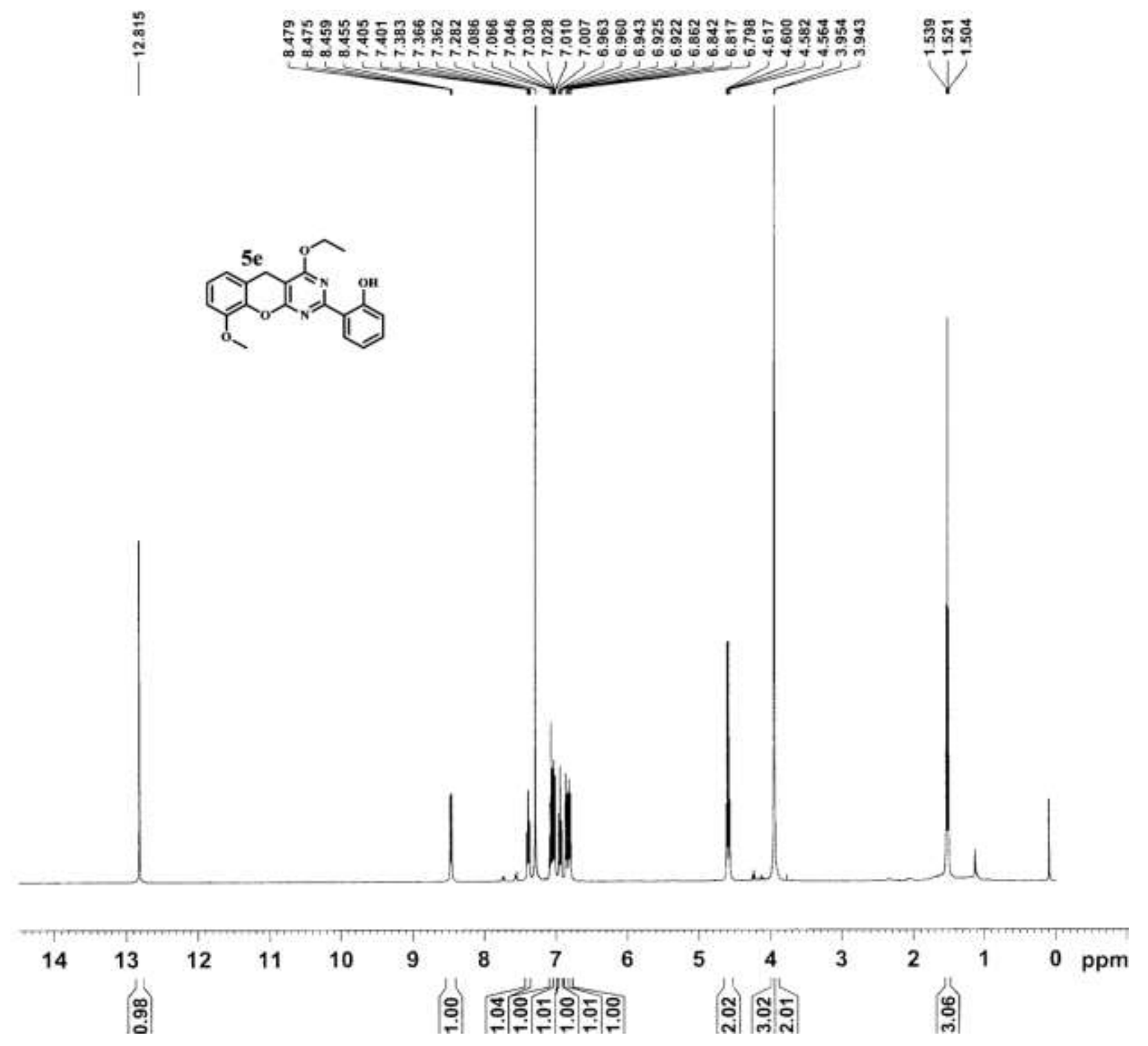




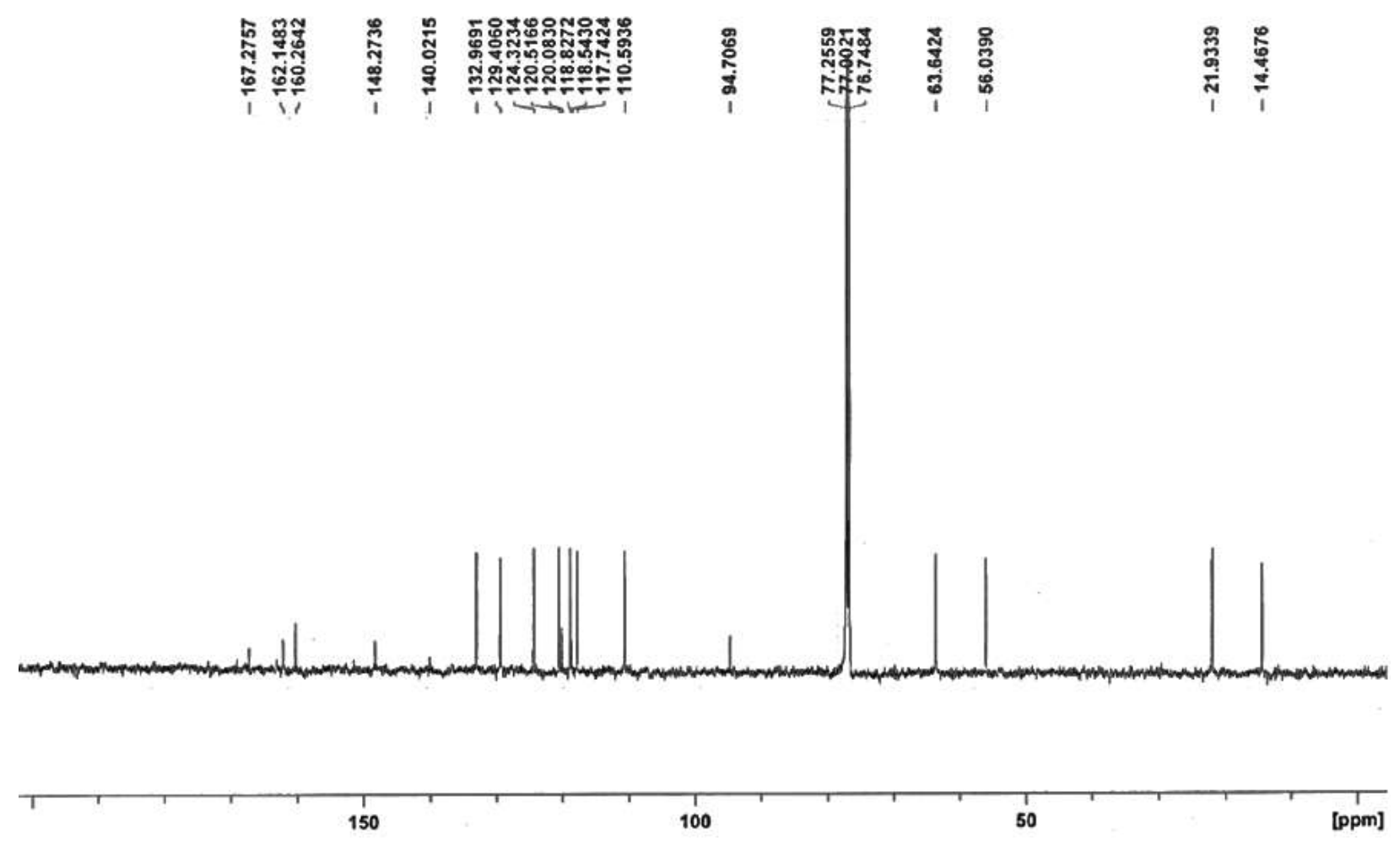




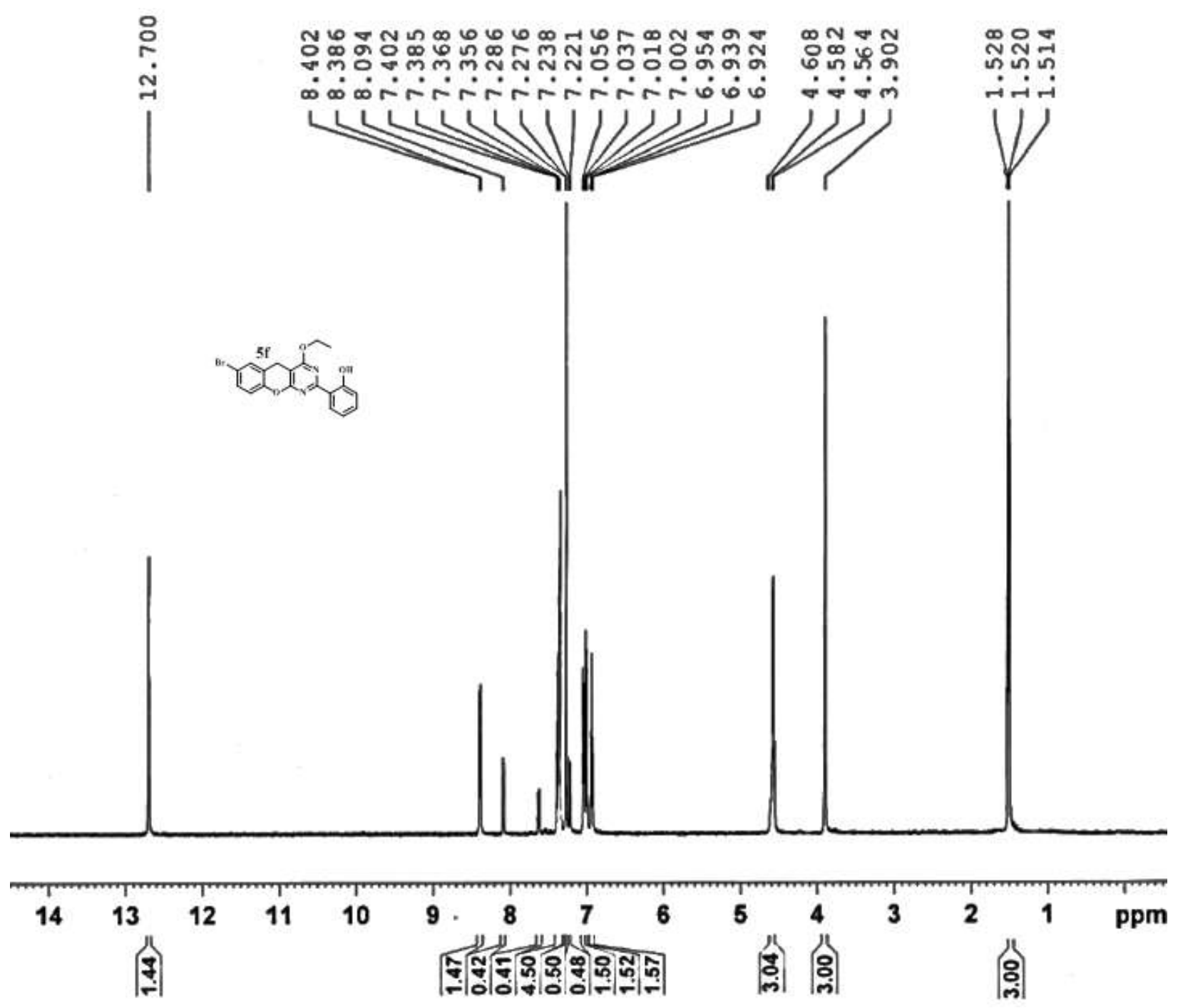

S24 


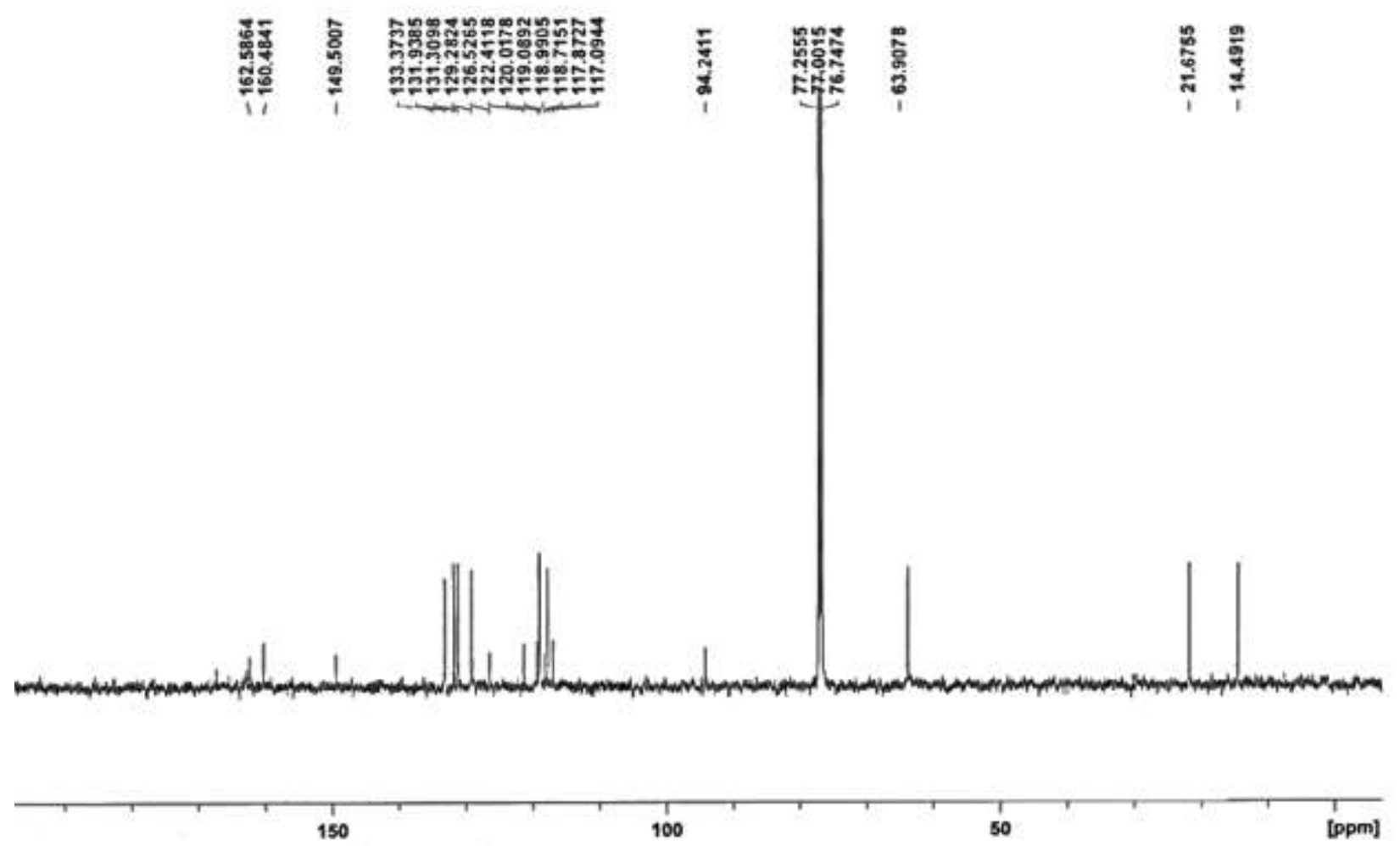




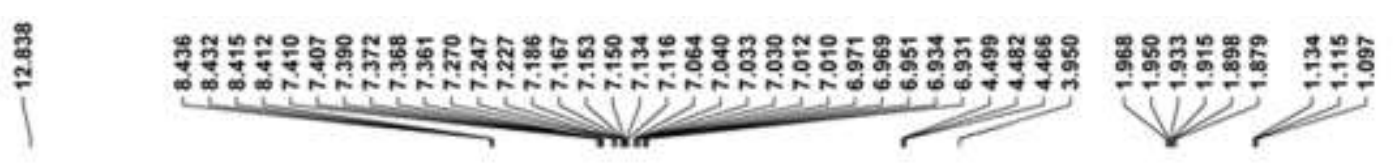<smiles>CCCOc1nc(-c2ccccc2C)nc2oc3ccccc3c(=S)c12</smiles>
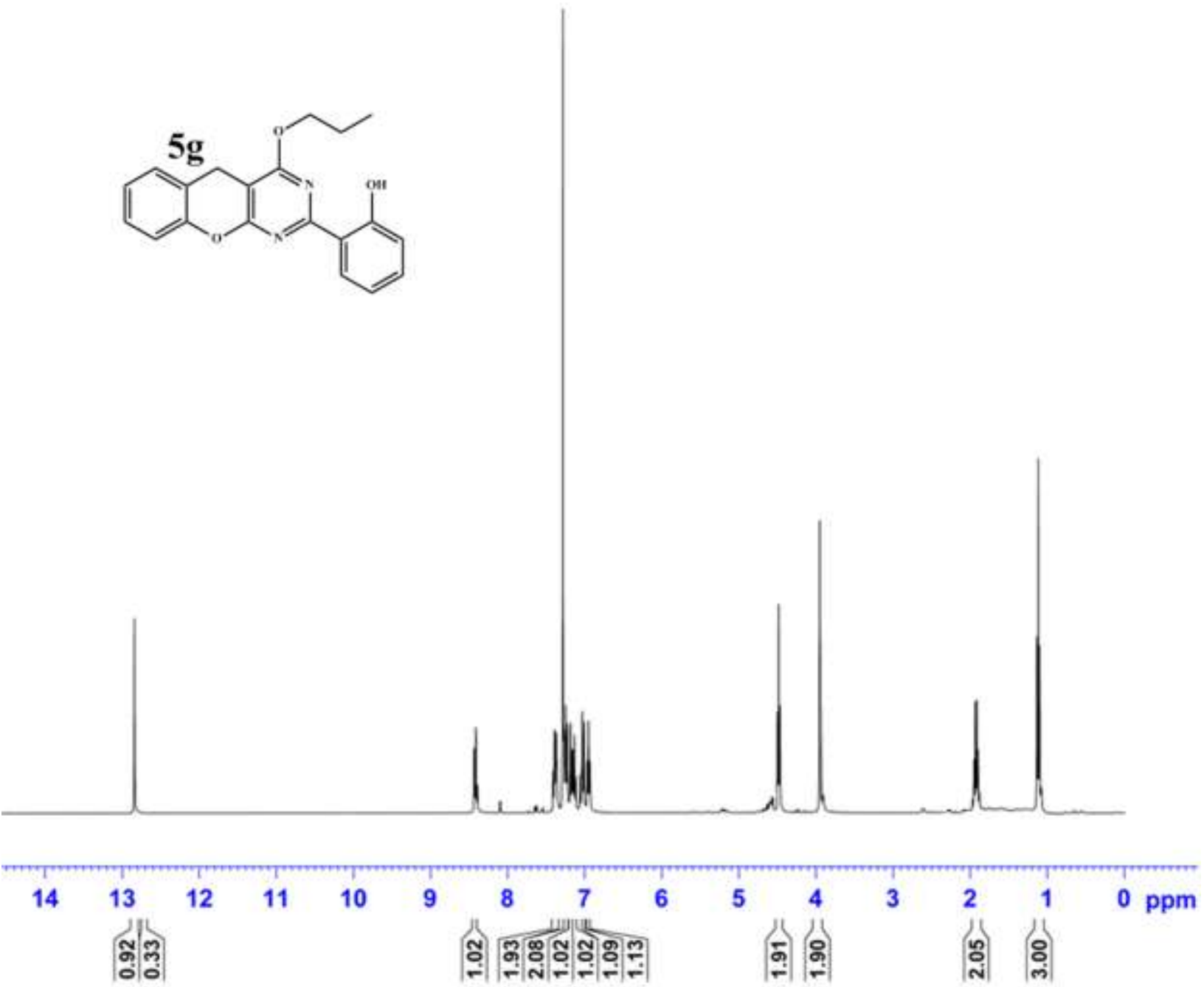


\begin{tabular}{|c|c|c|c|c|}
\hline 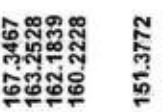 & 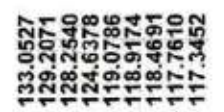 & $\begin{array}{l}\frac{9}{5} \\
\frac{5}{2}\end{array}$ & 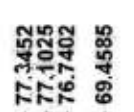 & 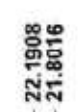 \\
\hline
\end{tabular}

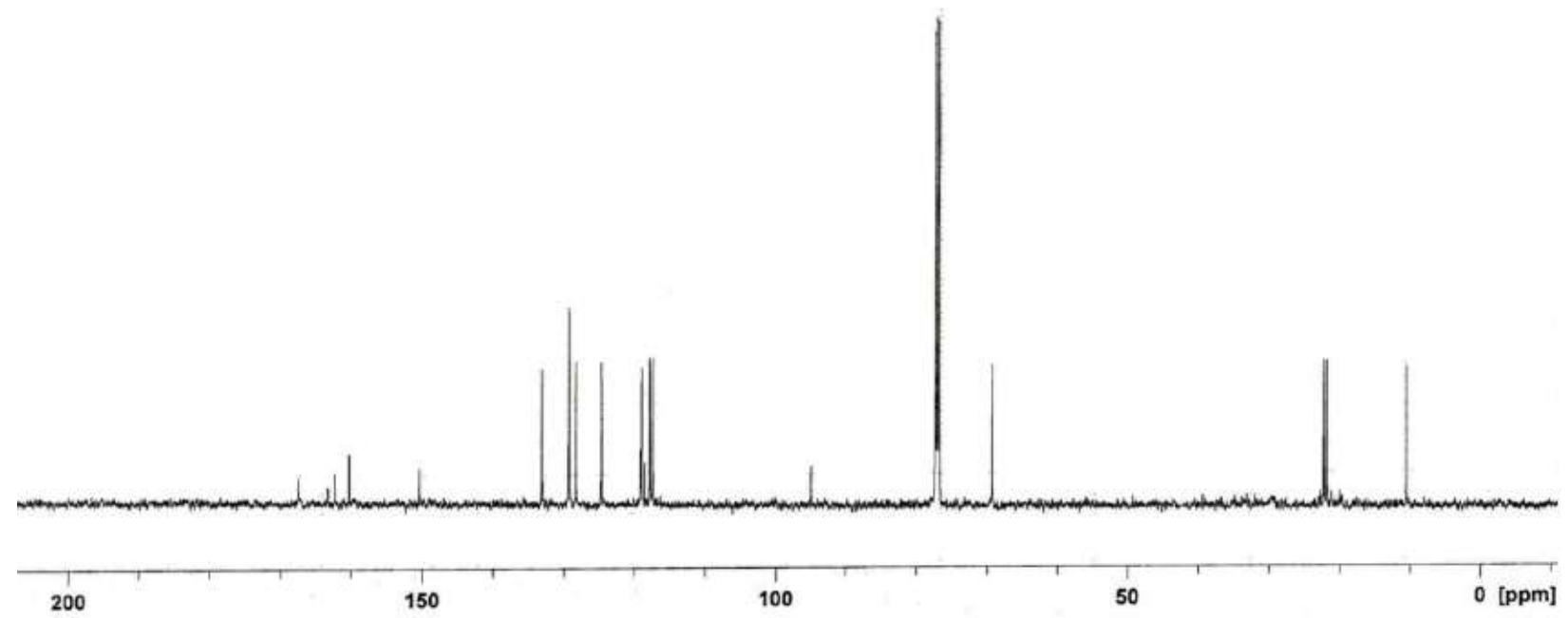




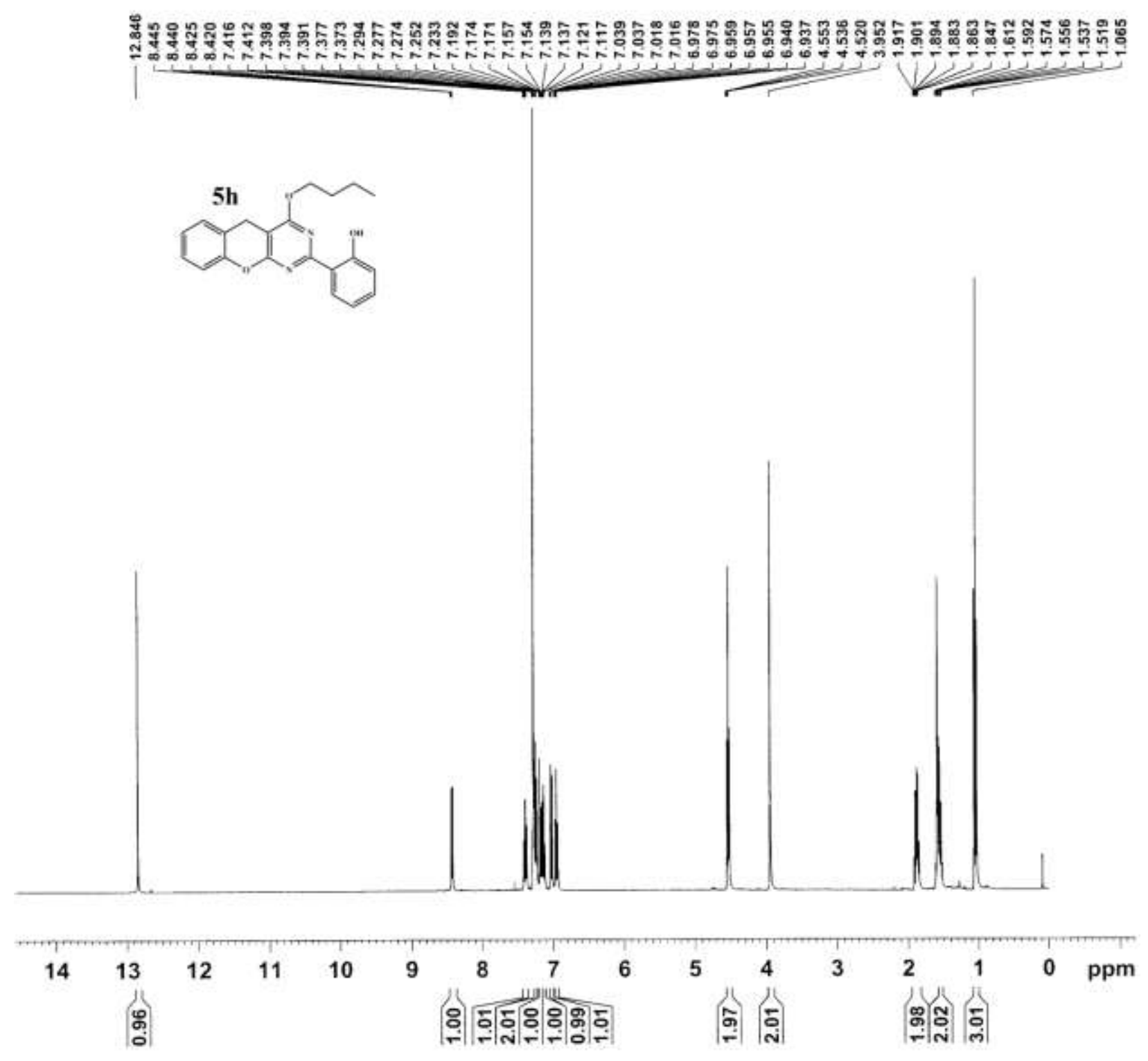




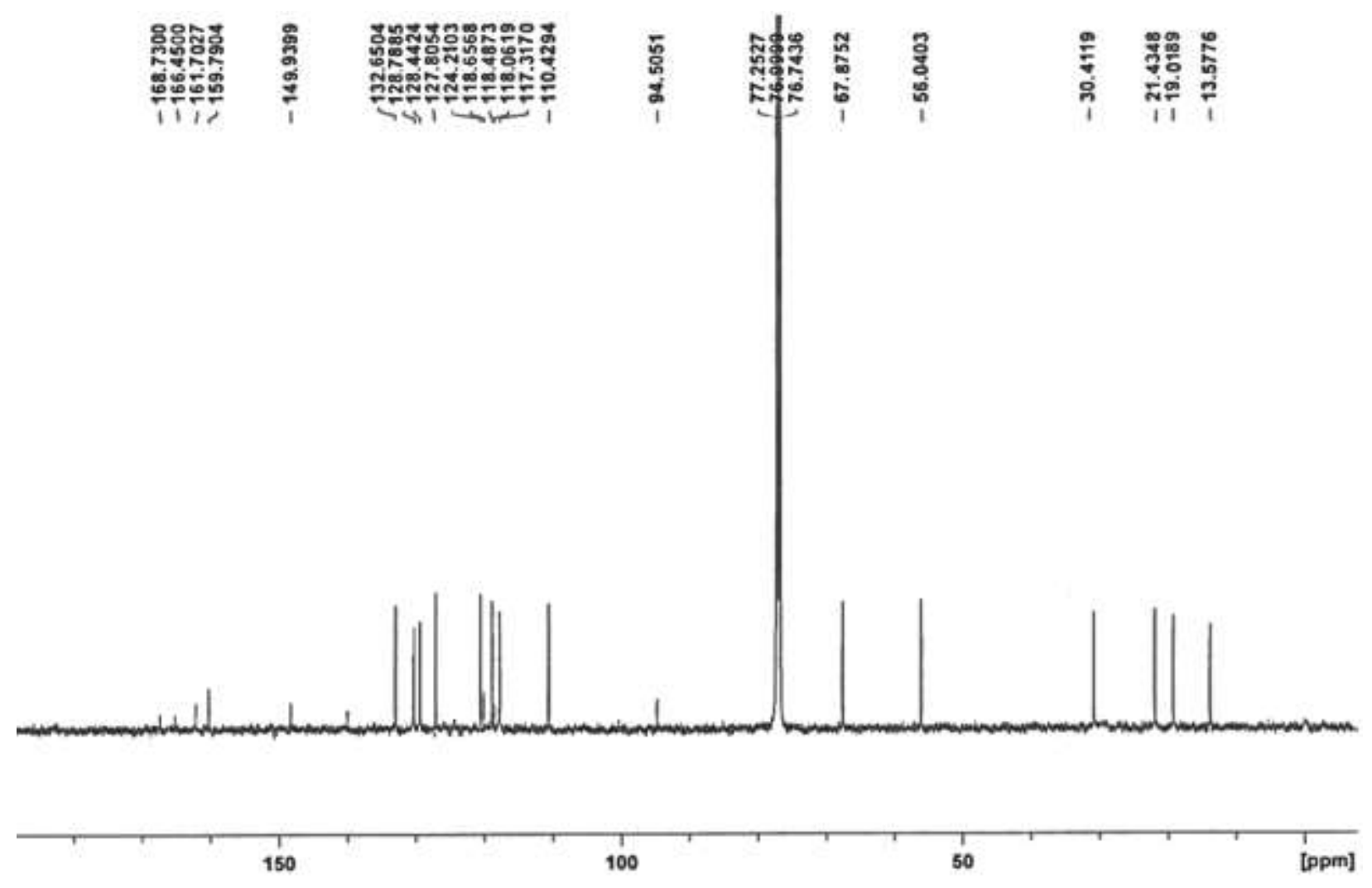




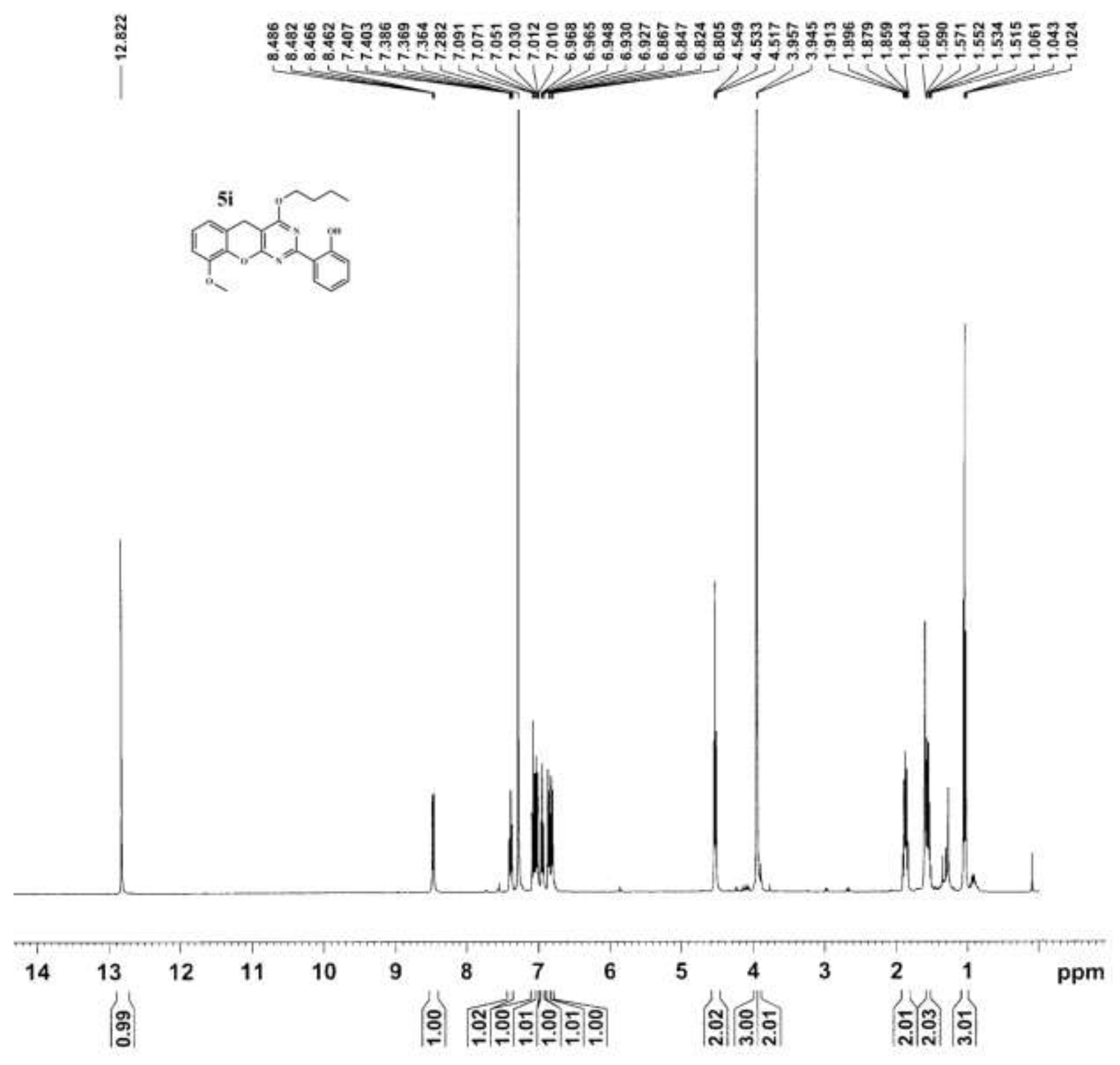




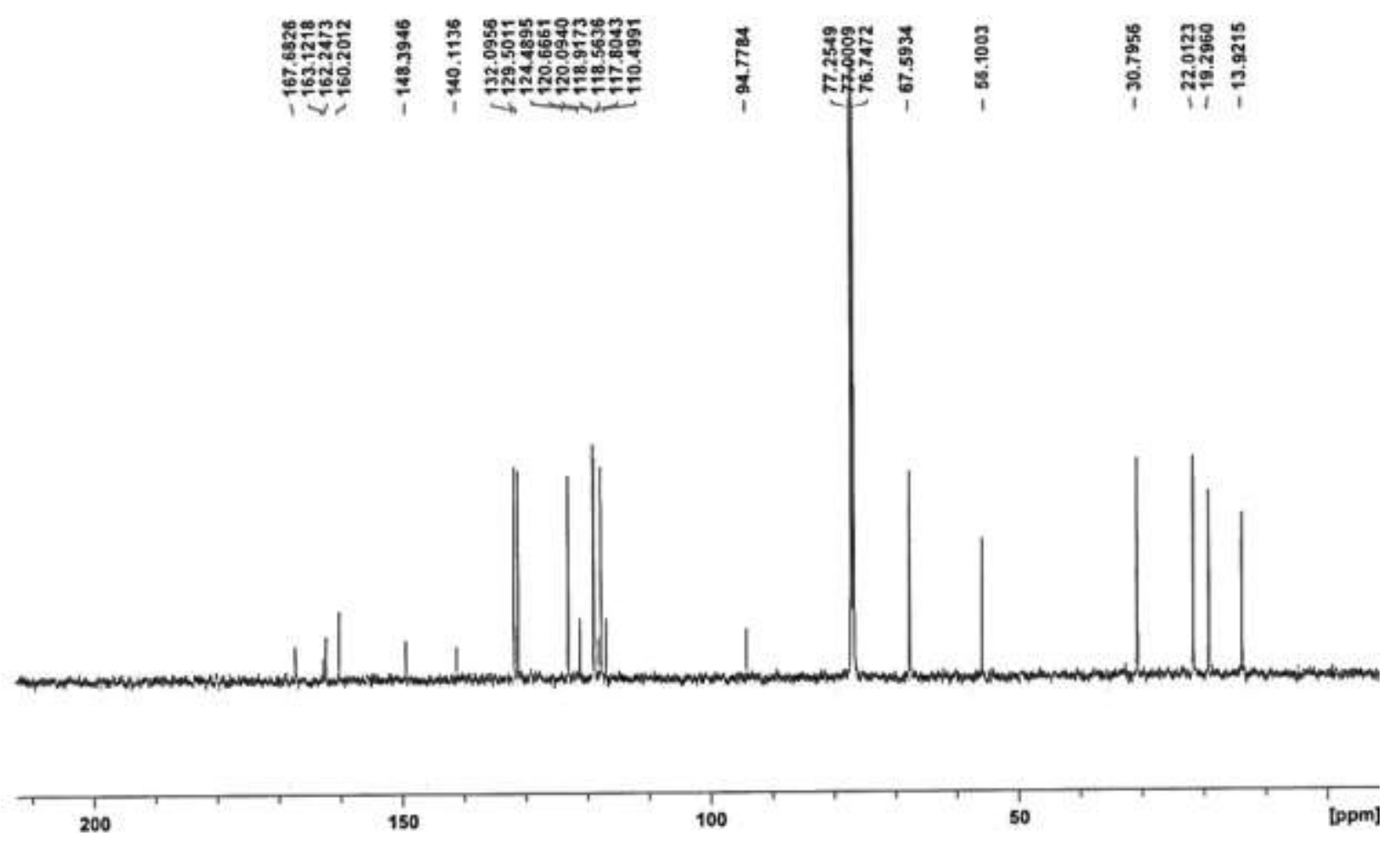



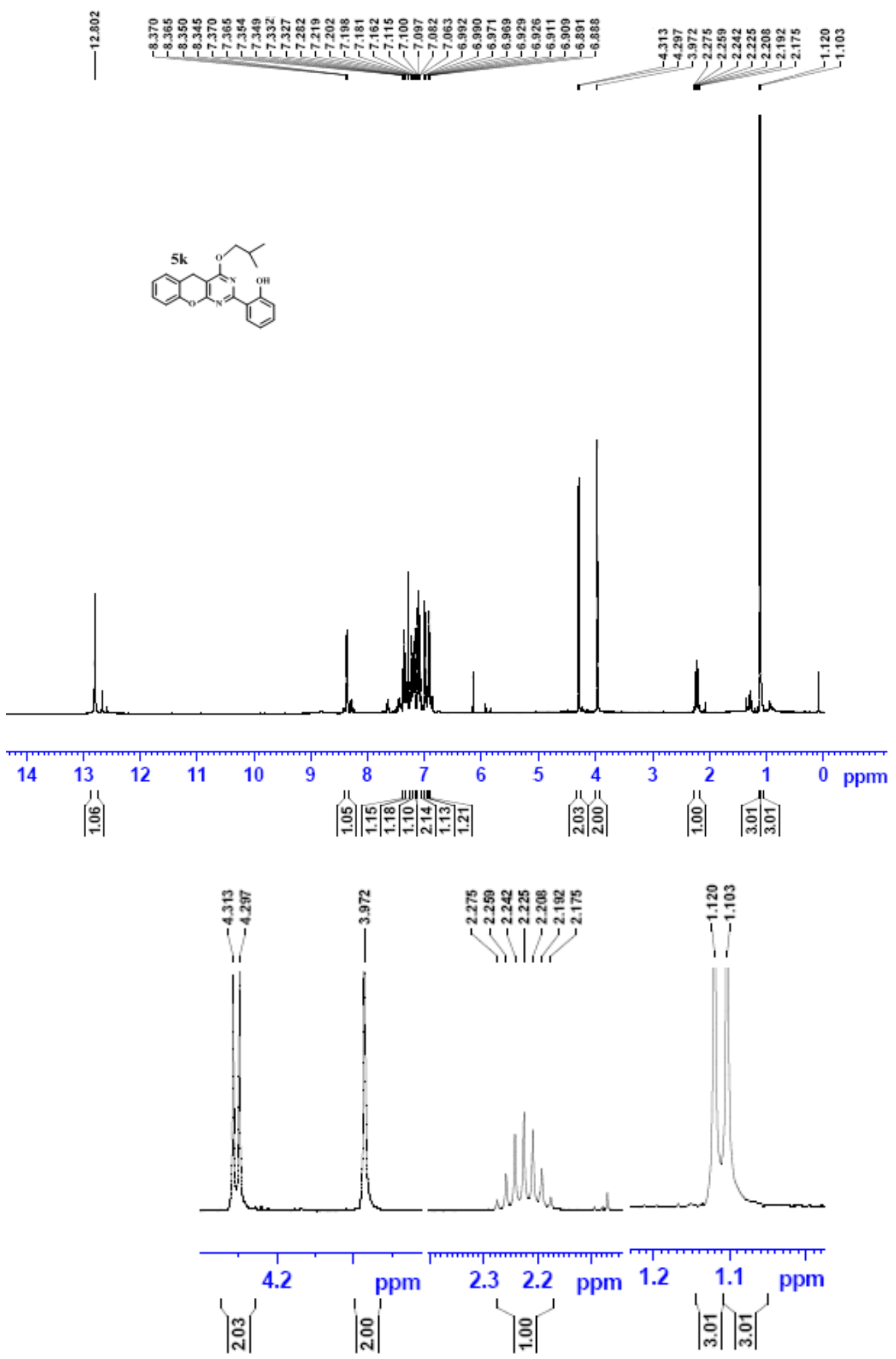

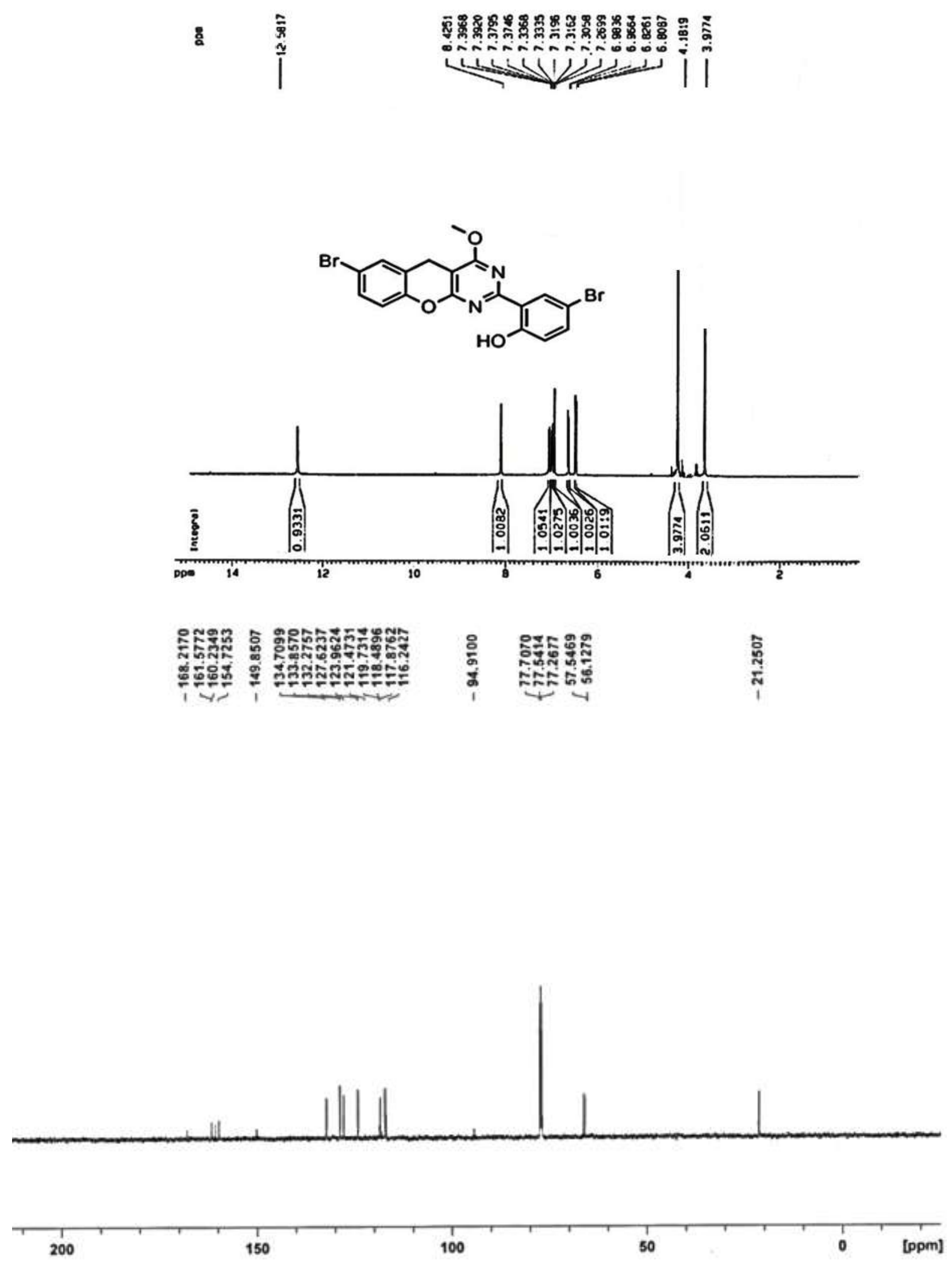


\section{References}

[1] (a) Falahati, M.; Ma'mani, L.; Saboury, A. A.; Shafiee, A.; Foroumadi, A.; Badiei A. R. Biochim. Biophys. Acta, 2011,1814, 1195-1202; (b) Kleitz, F.; Choi, S. H.; Ryoo, R. Chem. Commun., 2003, 17, 2136-2137.

[2] Punyacharoennon, P.; Charuchinda, S.; Srikulkit, K. J. Appl. Polym. Sci., 2008, 110, 3336-3347.

[3] Li, S.; Zhou, Z.; Abernathy, H.; Liu, M.; Li, W.; Ukai, J.; Hase, K.; Nakanishi, M. J. Mater. Chem., 2006, 16, 858-864.

[4] Zonouzi, A.; Hosseinzadeh, F.; Karimi, N.; Mirzazadeh R.; Ng, S. W. ACS Comb. Sci., 2013, 15, 240-246. 